\title{
Cognition-Oriented Treatments for Older Adults: a Systematic Overview of Systematic Reviews
}

\author{
Hanna Malmberg Gavelin ${ }^{1,2}$ (D) $\cdot$ Amit Lampit $^{2,3,4}$ (D) Harry Hallock ${ }^{3,4}$ (D) Julieta Sabatés $^{2}$ (D) $\cdot$ Alex Bahar-Fuchs $^{2}$ (D)
}

Received: 22 August 2019 / Accepted: 2 March 2020 / Published online: 7 April 2020

(C) The Author(s) 2020

\begin{abstract}
Cognition-oriented treatments - commonly categorized as cognitive training, cognitive rehabilitation and cognitive stimulation are promising approaches for the prevention of cognitive and functional decline in older adults. We conducted a systematic overview of meta-analyses investigating the efficacy of cognition-oriented treatments on cognitive and non-cognitive outcomes in older adults with or without cognitive impairment. Review quality was assessed by A Measurement Tool to Assess Systematic Reviews 2 (AMSTAR). We identified 51 eligible reviews, 46 of which were included in the quantitative synthesis. The confidence ratings were "moderate" for 9 (20\%), "low" for 13 (28\%) and "critically low" for 24 (52\%) of the 46 reviews. While most reviews provided pooled effect estimates for objective cognition, non-cognitive outcomes of potential relevance were more sparsely reported. The mean effect estimate on cognition was small for cognitive training in healthy older adults (mean Hedges' $g=0.32$, range $0.13-0.64,19$ reviews), mild cognitive impairment (mean Hedges' $g=0.40$, range $0.32-0.60$, five reviews), and dementia (mean Hedges' $g=0.38$, range $0.09-1.16$, seven reviews), and small for cognitive stimulation in dementia (mean Hedges' $g=0.36$, range $0.26-0.44$, five reviews). Meta-regression revealed that higher AMSTAR score was associated with larger effect estimates for cognitive outcomes. The available evidence supports the efficacy of cognition-oriented treatments improving cognitive performance in older adults. The extent to which such effects are of clinical value remains unclear, due to the scarcity of high-quality evidence and heterogeneity in reported findings. An important avenue for future trials is to include relevant non-cognitive outcomes in a more consistent way and, for meta-analyses in the field, there is a need for better adherence to methodological standards. PROSPERO registration number: CRD42018084490.
\end{abstract}

Keywords Cognitive training $\cdot$ Cognitive stimulation $\cdot$ Cognitive rehabilitation $\cdot$ Older adults $\cdot$ Systematic review $\cdot$ Overview

\section{Introduction}

As a larger proportion of the population reaches more advanced age, more people will be affected by cognitive aging.

Electronic supplementary material The online version of this article (https://doi.org/10.1007/s11065-020-09434-8) contains supplementary material, which is available to authorized users.

Hanna Malmberg Gavelin

hanna.malmberg-gavelin@umu.se

1 Department of Psychology, Umeå University, Umeå, Sweden

2 Academic Unit for Psychiatry of Old Age, University of Melbourne, Melbourne, Australia

3 Department of Neurology, Charité-Universitätsmedizin Berlin, Berlin, Germany

4 Berlin School of Mind and Brain, Humboldt-Universität zu Berlin, Berlin, Germany
Some cognitive decline occurs in what is usually considered normal ageing (Deary et al., 2009; Nyberg, Lovden, Riklund, Lindenberger, \& Backman, 2012), and it is a prominent feature in several predominantly age-related pathological conditions, including neurodegenerative and neurovascular diseases (Aarsland et al., 2017; Cumming, Marshall, \& Lazar, 2013; Weintraub, Wicklund, \& Salmon, 2012). Given the functional consequences of cognitive impairment in aging, and the subsequent personal, societal and financial costs, development of effective interventions that could maintain levels of cognitive functioning and delay cognitive and functional decline is a key priority in the field.

Cognition-oriented treatments is an umbrella term referring to several non-pharmacological treatment approaches which apply a range of techniques to engage thinking and cognition with various degrees of breadth and specificity. Unlike treatments that are primarily oriented towards outcomes that are behavioural (e.g. wandering), emotional (e.g. anxiety), or physical (e.g. sedentary lifestyle), in cognition-oriented 
treatments, the goals include improving or maintaining cognitive processes or addressing the impact of impairment in cognitive processes on associated functional ability in daily life (Bahar-Fuchs, Martyr, Goh, Sabates, \& Clare, 2019). Cognitive training involves repeated practice on a set of structured and standardized tasks, designed to target one or several cognitive abilities (Bahar-Fuchs, Clare, \& Woods, 2019; Clare $\&$ Woods, 2004). Cognitive stimulation consists of nonspecific engagement in a variety of activities aimed at general enhancement of cognitive and social functioning, usually carried out in group settings at clinics or residential care facilities (Woods, Aguirre, Spector, \& Orrell, 2012). Cognitive rehabilitation is an individualized approach with a functional emphasis, aimed at achieving or maintaining optimal levels of functioning in everyday life (Clare \& Woods, 2004; Kudlicka, Martyr, Bahar-Fuchs, Woods, \& Clare, 2019). Core elements of cognitive rehabilitation include identifying personallyrelevant goals and devising strategies for goal achievement in collaboration with the patients and their families (BaharFuchs et al., 2019). Thus, the focus of cognitive training is on restoring specific cognitive abilities, whereas cognitive stimulation is directed towards improving overall orientation and global cognitive status, and cognitive rehabilitation is focused on producing functional change in the everyday context (Bahar-Fuchs et al., 2019).

Given the potential of cognition-oriented treatments to maintain cognitive health in old age, the field has received widespread attention in recent years. Yet, the extent to which the three intervention approaches are efficacious on cognitive and non-cognitive outcomes in older adults, and whether such effects translate into prevention of further cognitive decline, remains hotly debated, as reflected in the highly publicised consensus letter (Stanford Center on Longevity, 2014) and counter letter (Cognitive Training Data, 2014) from the scientific community regarding cognitive training. Indeed, numerous systematic reviews and meta-analyses on these topics published in recent years have not produced clear and coherent evidence. Some of the contrasting findings from previous reviews are likely due to conceptual issues related to the various interventions, as well as differing methodological approaches by the review authors. Moreover, most metaanalyses have focused on one particular type of cognitionoriented treatment, group of older adults or outcome category. Therefore, there is a need to synthesize the available body of evidence, while also taking into account the methodological quality of published reviews.

In recent years, systematic overviews have emerged as a new form of evidence synthesis, allowing researchers to systematically identify and synthesize the available evidence from multiple systematic reviews on a given topic, resolve discrepancies and identify gaps in the literature $(\mathrm{H}$. Cooper \& Koenka, 2012; McKenzie \& Brennan, 2017). Thus, a systematic overview can address broader research synthesis questions than a single systematic review (H. Cooper \& Koenka, 2012). Such an approach to the efficacy of the three main types of cognition-oriented treatments on cognitive and non-cognitive outcomes for older adults on the spectrum from cognitive health to dementia could be of value for both research and practice and provide future directions for the field. This overview provides researchers and clinicians alike with a comprehensive and accessible summary of the available evidence, while also using a rigorous quality assessment process to evaluate the methodological quality of published metaanalyses, and explore its relation to review outcomes. This approach could therefore address some of the previous discrepancies in the field and identify areas in which further high-quality research is needed.

The aim of the present overview is to synthesize systematic reviews with meta-analysis of cognition-oriented treatments in older adults (aged $>50$ years). We sought to (1) provide an overview of the available evidence on the efficacy of the three main types of cognition-oriented treatments for older adults with or without cognitive decline on cognitive and non-cognitive outcomes, (2) explore the outcomes of reviews in relation to their methodological quality, (3) evaluate the strength and quality of the evidence for cognition-oriented treatments in older adults and, (4) suggest recommendations for further research and research synthesis in the field.

\section{Methods}

\section{Protocol and Registration}

This review follows the Preferred Reporting Items of Systematic Reviews and Meta-analyses (PRISMA) guidelines (Liberati et al., 2009) and the study protocol was prospectively registered with PROSPERO (CRD42018084490).

\section{Search Strategy and Study Selection}

An electronic database search of MEDLINE, EMBASE, PsychINFO and Cochrane Database of Systematic Reviews was conducted from inception to April 2019 to identify systematic reviews with meta-analysis examining the effects of cognition-oriented treatments on cognitive or non-cognitive outcomes for older adults with or without cognitive decline (see Supplementary Material 1 for the full search strategy). No restrictions on language or publication type were applied. Initial screening of titles and abstracts was conducted by one reviewer (HMG) and full-text screening of potentially relevant papers was conducted independently by two reviewers (HMG and $\mathrm{ABF}, \mathrm{AL}$ or JS). Disagreements were resolved by consensus and involvement of a third reviewer if necessary. The electronic search was complemented by hand-searching the references of retrieved articles. 


\section{Eligibility Criteria}

Type of studies. Systematic reviews using meta-analytic methods including randomized or non-randomized controlled trials were included. Reviews that included non-controlled trials were included if the results from controlled trials were reported separately. Overviews including a combination of reviews and primary trials were excluded.

Types of participants. Reviews were included if they focused on older adults (mean age $>50$ years), with or without cognitive decline. This included cognitively unimpaired older adults (hereby referred to as "healthy older adults"), as well as conditions that are associated with cognitive impairment in older adults, such as mild cognitive impairment (MCI), dementia (irrespective of aetiology), Parkinson's disease (PD) and stroke. Conditions that may be associated with impaired cognitive functioning but are not specific to older adults such as depression and traumatic brain injury were included if it was clear from the review that it specifically addressed older adults. Reviews covering a population not restricted to older adults were included if the results for older adults were reported separately.

Types of interventions. Reviews were included if they focused on interventions that could be classified as cognitive training, cognitive rehabilitation or cognitive stimulation, according to the definitions provided by Clare and Woods (2004). Reviews focusing largely on combined interventions such as combined cognitive and aerobic training were excluded.

Types of comparisons. Reviews were included if the primary trials used an active (e.g. placebo treatment) or passive (e.g. treatment-as-usual, wait-list, no treatment) control condition. Reviews comparing cognition-oriented treatments with other interventions, such as physical exercise, or comparing different types of cognition-oriented treatments were excluded.

Types of outcomes. Outcomes included objective cognitive function (global and domain-specific), subjective cognitive function, psychosocial function (e.g. quality of life, mood, depression), functional abilities (activities of daily living), caregiver burden (e.g. burden of care, caregiver stress, caregiver depression), clinical status (e.g. clinical severity and progression or stability rates, rates of admission to residential care, discharge destination) and behavioural and psychological symptoms of dementia. Long-term outcomes were excluded.

\section{Data Collection and Coding}

Data collection was conducted independently by two reviewers (HMG and JS or HH). Disagreements were resolved by consensus and by the involvement of a senior reviewer (AL or $\mathrm{ABF}$ ) if necessary. For each review, the following data was extracted: review characteristics, details of search strategy and meta-analytic methods, coding of interventions, populations and outcomes, and recording of effect sizes and confidence intervals for each reported outcome. When reviews included a mixture of populations or interventions, we classified them as belonging to the category to which $>75 \%$ of the included primary trials adhered to. If such a categorization was not possible, they were coded as "mixed population" or "mixed cognition-oriented treatment". Effect sizes were extracted if they were reported as between-group mean differences and if there was a minimum of two primary trials contributing to the effect size estimate. Reviews that did not provide sufficient statistical information (i.e., between-group effect size estimates and associated confidence intervals) were synthesized narratively.

\section{Quality Assessment}

Quality assessment was conducted independently by two authors (HMG and HH or JS) using A Measurement Tool to Assess Systematic Reviews 2 (AMSTAR: Shea et al., 2017) and disagreements were solved by consensus or by consulting a senior author (ABF or $\mathrm{AL}$ ). In the case that an author of the present overview had co-authored an eligible review, they were not involved in the quality assessment of that review. In line with the recommendations by Shea et al. (2017), domains in which weaknesses would critically diminish the validity of a review were identified. The following items were considered critical: Item 4 - adequacy of the literature search, Item 9 - adequate assessment of risk of bias, Item 11- appropriateness of meta-analytic methods, Item 13 - consideration of risk of bias when interpreting the results of the review and, Item 15 - assessment of presence and likely impact of publication bias. Based on the fulfilment of critical and non-critical items, the overall confidence in the results of each review was rated as following (a) high - no or one non-critical weakness, (b) moderate - more than one noncritical weakness, (c) low - one critical flaw with or without non-critical weaknesses, or (d) critically low - more than one critical flaw with or without non-critical weaknesses.

In addition, a total score $(0-16)$ was applied for each review. One point was given for each AMSTAR item if the review met the answer "Yes", 0.5 point for "Partial Yes" and 0 points for "No". We made some minor adjustments to the AMSTAR ratings as follow. For Item 2 we excluded the item relating to "justification for any deviations from the protocol." For Item 4, adherence to "justified publication restriction (e.g., language)" was only required for a full "Yes" and for Item 11, adherence to "justified combining the data in a meta-analysis" was only required if any contra-indication for meta-analysis existed. 


\section{Data Analysis}

Analyses were performed using Comprehensive MetaAnalysis version 3 (Biostat, NJ). For each reported outcome, the between-group mean difference or standardized mean difference and associated confidence interval (CI) was entered into the Comprehensive Meta-Analysis software. To yield unified effect size estimates, we recalculated all effect sizes into Hedges' $g$ with a 95\% CI. The results were investigated by outcome domain (objective cognitive function, subjective cognitive function, psychosocial function, functional abilities, caregiver outcomes, clinical outcomes and behavioural and psychological symptoms of dementia). When reviews provided more than one effect size estimate per outcome domain, the standardized mean difference and variance for each outcome was combined into a single composite. If a review reported an overall composite measure as well as separate results for different subdomains, the composite measure was preferred. The standardized mean differences for each of the main outcome categories were summarized graphically to provide an overview of the available evidence across the different populations, interventions and outcomes. As stated in the registered protocol, we initially intended to pool the data in a secondorder meta-analysis if a sufficient number of reviews were available for a certain population and outcome category. However, to avoid the problem of overlapping primary trials across reviews (H. Cooper \& Koenka, 2012; McKenzie \& Brennan, 2017), we decided against this approach. Instead, for each outcome category, when there was a minimum of three meta-analyses available for a given population or intervention combination, we calculated the mean standardized mean difference and compared this to the standardized mean difference of the "optimal review", defined as the review that met as many of the following characteristics as possible (1) recent (last 5 years), (2) comprehensive (likely to include most of the relevant literature at the time of publication), (3) rigorous (moderate confidence rating or AMSTAR score $>10$ ). If the mean of the standardized mean differences and the standardized mean difference from the optimal review was reasonably similar, we regarded this as a good approximation of the effect of the intervention on the population and outcome. Using established convention, an effect size of less than 0.2 was considered negligible, between 0.2 and 0.5 small, between 0.5 and 0.8 medium and more than 0.8 large. A sensitivity analysis was also conducted by calculating the mean standardized mean difference while excluding reviews that had a critically low confidence rating. This was done for the main analysis of objective cognitive outcomes, when there was a minimum of three meta-analyses available.

Spearman's rank correlation was used to investigate the association between AMSTAR score and year of publication. In addition, independent samples $t$-tests were used to compare the mean AMSTAR score of reviews that included only randomized controlled trials (RCTs) versus those that included both RCTs and non-RCTs, and of reviews that had a preregistered protocol versus those that did not. These analyses were performed using IBM SPSS Statistics version 24.

Finally, restricted likelihood, three-level meta-regression models were used to investigate the moderating effect of AMSTAR score, year of publication and number of included studies on overall effect estimate on objective cognitive outcomes. Given many of the included reviews provided more than one effect estimate per outcome for analysis, three-level meta-regression analyses assessed the extent to which the model explains heterogeneity within $\left(\tau_{(2)}^{2}\right)$ and between studies $\left(\tau_{(3)}^{2}\right)$, expressed as $R_{(2)}^{2}$ and $R_{(3)}^{2}$, respectively (Cheung, 2019). Analyses were conducted using the packages metafor (Viechtbauer, 2010) and metaSEM (Cheung, 2014) on R 3.6.0.

\section{Results}

\section{Study Selection}

After duplicate search results were removed, 2128 records were initially screened for eligibility, out of which 1972 were excluded based on titles and abstracts. Of the 156 articles assessed in the full-text screening, 105 were excluded. The most common reason for exclusion was that the review did not include a meta-analysis, followed by not covering the target population or intervention (see Supplementary Material 2 for the full reasons for exclusion). A total of 51 meta-analyses fulfilled inclusion criteria (Fig. 1).

Three reviews (Karbach \& Verhaeghen, 2014; Verhaeghen, Marcoen, \& Goossens, 1992; Wilson, 2008) did not report effect size estimates as between-group differences, one (Sitzer, Twamley, \& Jeste, 2006) did not provide sufficient information for calculation of the CI of the reported effect sizes, and one (Olazaran et al., 2010) reported treatment recommendations. These reviews were therefore only summarized narratively (see Supplementary Material 3). Henceforth, results are presented based on the 46 meta-analyses that provided sufficient information to be included in the quantitative synthesis. A narrative summary of these reviews is provided in Supplementary Material 4.

\section{Characteristics of Included Studies}

The characteristics and methods of the included meta-analyses are presented in Table 1 and Table 2. Out of 46 reviews, 34 reported meta-analytic results for cognitive training, three reported meta-analytic results for cognitive stimulation, three included both cognitive training and cognitive stimulation and six were classified as mixed cognition-oriented 


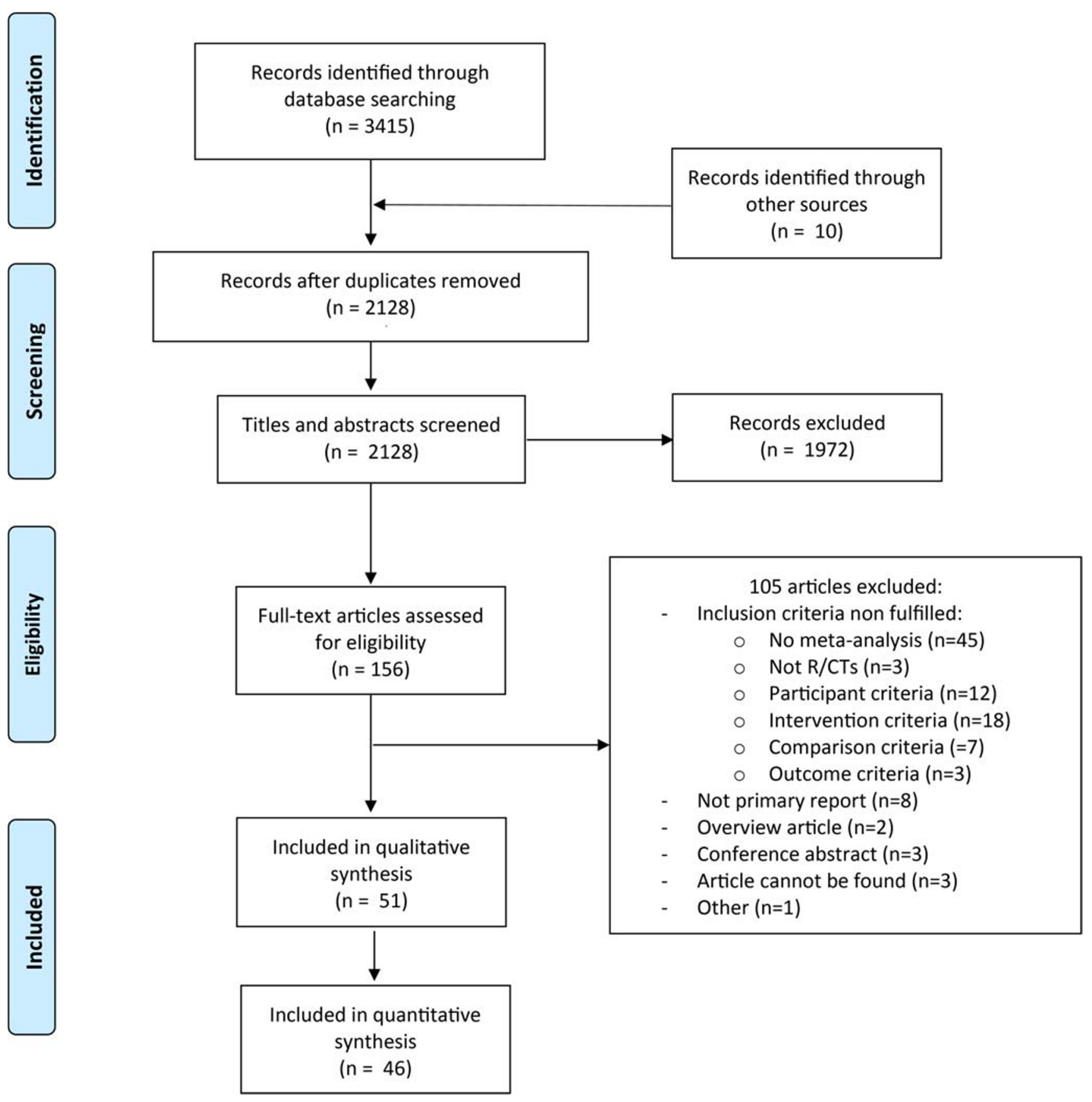

Fig. 1 PRISMA flow chart

treatments. No review reported meta-analytic results for cognitive rehabilitation. A summary of the interventions, populations and outcomes covered by the included meta-analyses is provided in Table 3. Some reviews reported meta-analytic results for more than one population or intervention, which is described in more detail below.

Type of studies. 32 reviews included only RCTs and 14 reviews included a combination of RCTs and non-RCTs.

Populations. Seventeen reviews reported meta-analytic results for healthy older adults (Table 1), three of which focused specifically on individuals with subjective cognitive complaints (Bhome, Berry, Huntley, \& Howard, 2018; Metternich, Kosch, Kriston, Harter, \& Hull, 2010; Smart et al., 2017). Four reviews focused on individuals with MCI (Chandler, Parks, Marsiske, Rotblatt, \& Smith, 2016; Gates et al., 2019; Sherman, Mauser, Nuno, \& Sherzai, 2017; Wang et al., 2014). Ten reviews focused on people with dementia (Alves et al., 2013; Bahar-Fuchs et al., 2019; C. Cooper et al., 2012; Folkerts, Roheger, Franklin, Middelstadt, \& Kalbe, 2017; Huntley, Gould, Liu, Smith, \& Howard, 2015; Kim et al., 2017; Lee et al., 2019; P. Leung, Orgeta, \& Orrell, 2017; Song, Lee, \& Song, 2016; Woods et al., 2012). One 
Table 1 Characteristics of Included Meta-Analyses

\begin{tabular}{|c|c|c|c|c|c|c|c|}
\hline Study & $\begin{array}{l}\text { Populations } \\
\text { included in current } \\
\text { analyses }\end{array}$ & $\begin{array}{l}\text { Interventions } \\
\text { included in } \\
\text { current analyses }\end{array}$ & $\begin{array}{l}\text { Pre-specified control } \\
\text { conditions }\end{array}$ & $\begin{array}{l}\text { Outcomes included in } \\
\text { current analyses }\end{array}$ & $\begin{array}{l}\text { No. of } \\
\text { studies }\end{array}$ & $\begin{array}{l}\text { No. of } \\
\text { participants }\end{array}$ & $\begin{array}{l}\text { Age of } \\
\text { participants }\end{array}$ \\
\hline $\begin{array}{l}\text { Alves et al. } \\
\quad(2013)\end{array}$ & Dementia (AD) & $\mathrm{CT}$ & Active & $\begin{array}{l}\text { Attention and } \\
\text { concentration } \\
\text { Delayed } \\
\text { auditory/verbal } \\
\text { memory } \\
\text { Delayed verbal } \\
\text { recognition } \\
\text { Delayed visuospatial } \\
\text { memory } \\
\text { Immediate } \\
\text { auditory/verbal } \\
\text { memory } \\
\text { Immediate visuospatial } \\
\text { memory } \\
\text { Global cognition } \\
\text { (screening) } \\
\text { Verbal fluency } \\
\text { phonemic } \\
\text { Verbal fluency } \\
\text { semantic }\end{array}$ & 4 & 133 & Not reported \\
\hline $\begin{array}{l}\text { Bahar-Fuchs } \\
\text { et al. (2019) }\end{array}$ & $\begin{array}{l}\text { Dementia (mild to } \\
\text { moderate, any } \\
\text { subtype) }\end{array}$ & $\mathrm{CT}$ & $\begin{array}{l}\text { Wait-list, no } \\
\text { treatment/standard } \\
\text { treatment, active control } \\
\text { or alternative treatment } \\
\text { (not included in current } \\
\text { analyses) }\end{array}$ & $\begin{array}{l}\text { Global cognition } \\
\text { (composite) } \\
\text { Metacognition } \\
\quad \text { (informant-reported) } \\
\text { Metacognition } \\
\quad \text { (self-reported) } \\
\text { General health and } \\
\text { QoL } \\
\text { Mood } \\
\text { ADL } \\
\text { Burden of care } \\
\text { Disease progression } \\
\text { BPSD }\end{array}$ & 33 & 1926 & Not reported \\
\hline $\begin{array}{l}\text { Bhome et al. } \\
\text { (2018) }\end{array}$ & $\begin{array}{l}\text { Healthy OA (with } \\
\text { subjective } \\
\text { cognitive } \\
\text { decline) }\end{array}$ & $\mathrm{CT}$ & Active and non-active & $\begin{array}{l}\text { Global cognition } \\
\text { (composite) } \\
\text { Metacognition } \\
\text { Psychological } \\
\text { well-being }\end{array}$ & $\begin{array}{r}20(11 \\
\text { CT })\end{array}$ & $\begin{array}{l}\text { Not } \\
\text { reported }\end{array}$ & Not reported \\
\hline $\begin{array}{l}\text { Chandler et al. } \\
\text { (2016) }\end{array}$ & MCI & Mixed COT & Not reported & $\begin{array}{l}\text { Metacognition } \\
\text { Mood } \\
\text { QoL } \\
\text { ADL }\end{array}$ & 30 & 2093 & Not reported \\
\hline $\begin{array}{l}\text { Chiu et al. } \\
\text { (2017) }\end{array}$ & Healthy OA & $\mathrm{CT}$ & Any form of control group & $\begin{array}{l}\text { Attention } \\
\text { Executive function } \\
\text { Global cognition } \\
\quad \text { (screening) } \\
\text { Memory } \\
\text { Visuospatial ability }\end{array}$ & 31 & 6003 & $65.1-85.1$ \\
\hline $\begin{array}{l}\text { Cooper et al. } \\
\text { (2012) }\end{array}$ & Dementia & $\mathrm{CS}$ & Not reported & QoL & $\begin{array}{c}20(3 \\
\text { CS })\end{array}$ & 1769 (total) & Not reported \\
\hline $\begin{array}{l}\text { das Nair et al. } \\
\quad(2016)\end{array}$ & $\begin{array}{l}\text { Stroke (with } \\
\text { memory deficits) }\end{array}$ & $\begin{array}{l}\mathrm{CT} \text { (focusing on } \\
\text { memory } \\
\text { deficits) }\end{array}$ & $\begin{array}{l}\text { Alternative form of } \\
\text { treatment or no memory } \\
\text { intervention }\end{array}$ & $\begin{array}{l}\text { Comprehensive } \\
\text { memory batteries } \\
\text { Verbal memory }\end{array}$ & 13 & 514 & $31-68$ \\
\hline
\end{tabular}


Table 1 (continued)

\begin{tabular}{|c|c|c|c|c|c|c|c|}
\hline Study & $\begin{array}{l}\text { Populations } \\
\text { included in current } \\
\text { analyses }\end{array}$ & $\begin{array}{l}\text { Interventions } \\
\text { included in } \\
\text { current analyses }\end{array}$ & $\begin{array}{l}\text { Pre-specified control } \\
\text { conditions }\end{array}$ & $\begin{array}{l}\text { Outcomes included in } \\
\text { current analyses }\end{array}$ & $\begin{array}{l}\text { No. of } \\
\text { studies }\end{array}$ & $\begin{array}{l}\text { No. of } \\
\text { participants }\end{array}$ & $\begin{array}{l}\text { Age of } \\
\text { participants }\end{array}$ \\
\hline & & & & $\begin{array}{l}\text { Subjective memory } \\
\text { Mood } \\
\text { Functional ability }\end{array}$ & & & \\
\hline $\begin{array}{l}\text { Floyd and } \\
\text { Scogin } \\
(1997)\end{array}$ & $\begin{array}{l}\text { Mixed (older adults } \\
\text { aged } 60 \text { and } \\
\text { above, without } \\
\text { dementia) }\end{array}$ & $\begin{array}{l}\text { CT (memory } \\
\text { training) }\end{array}$ & Not reported & $\begin{array}{l}\text { Subjective memory } \\
\text { Depression }\end{array}$ & 27 & 1150 & 70.6 \\
\hline $\begin{array}{l}\text { Folkerts et al. } \\
\qquad(2017)\end{array}$ & $\begin{array}{l}\text { Dementia (living in } \\
\text { long-term care } \\
\text { facilities) }\end{array}$ & $\mathrm{CS}$ and $\mathrm{CT}$ & $\begin{array}{l}\text { Usual care, waiting list or } \\
\text { active control }\end{array}$ & $\begin{array}{l}\text { Global cognition } \\
\text { (screening) } \\
\text { QoL/well-being }\end{array}$ & 27 & 1341 & $69.8-87.8$ \\
\hline $\begin{array}{l}\text { Gates, Rutjes, } \\
\text { et al. (2019) }\end{array}$ & Healthy OA & $\begin{array}{l}\text { CT } \\
\text { (computerized) }\end{array}$ & $\begin{array}{l}\text { Active (unguided } \\
\text { computer/screen-based } \\
\text { tasks), or inactive (no } \\
\text { intervention expected to } \\
\text { have an effect on cog- } \\
\text { nition) }\end{array}$ & $\begin{array}{l}\text { Episodic memory } \\
\text { Executive function } \\
\text { Global cognition } \\
\quad \text { (composite) } \\
\text { Speed of processing } \\
\text { Working memory }\end{array}$ & 8 & 1183 & $67-82$ \\
\hline $\begin{array}{l}\text { Gates, } \\
\text { Vernooij, } \\
\text { et al. (2019) }\end{array}$ & MCI & $\begin{array}{l}\text { CT } \\
\text { (computerized) }\end{array}$ & $\begin{array}{l}\text { Active (unguided } \\
\text { computer/screen-based } \\
\text { tasks), or inactive (no } \\
\text { intervention expected to } \\
\text { have an effect on cog- } \\
\text { nition) }\end{array}$ & $\begin{array}{l}\text { Episodic memory } \\
\text { Executive function } \\
\text { Global cognition } \\
\quad \text { (screening) } \\
\text { Speed of processing } \\
\text { Verbal fluency } \\
\text { Working memory } \\
\text { Depression } \\
\text { Functional } \\
\text { performance }\end{array}$ & 8 & 660 & $70-82$ \\
\hline $\begin{array}{l}\text { Gross et al. } \\
\quad(2012)\end{array}$ & Healthy OA & $\begin{array}{l}\mathrm{CT} \text { (memory } \\
\text { training) }\end{array}$ & Not reported & Memory & 35 & 3797 & $60-98$ \\
\hline $\begin{array}{r}\text { Hill et al. } \\
\text { (2017) }\end{array}$ & $\begin{array}{l}\text { MCI and dementia } \\
\text { (any ethology) }\end{array}$ & $\begin{array}{l}\text { CT } \\
\text { (computerized) }\end{array}$ & Active or passive & $\begin{array}{l}\text { Global cognition } \\
\text { (composite) } \\
\text { Psychosocial } \\
\quad \text { functioning } \\
\text { ADL } \\
\text { ADL instrumental }\end{array}$ & $\begin{array}{l}17 \text { MCI } \\
12 \\
\text { de- } \\
\text { menti- } \\
\text { a }\end{array}$ & $\begin{array}{l}686 \mathrm{MCI} \\
389 \\
\text { demen- } \\
\text { tia }\end{array}$ & $\begin{array}{l}\text { 67-81 MCI } \\
66-81 \\
\quad \text { dementia }\end{array}$ \\
\hline $\begin{array}{l}\text { Hindin and } \\
\text { Zelinski } \\
\text { (2012) }\end{array}$ & Healthy OA & CT & Not reported & $\begin{array}{l}\text { Global cognition } \\
\text { (composite) }\end{array}$ & $\begin{array}{r}42(25 \\
\mathrm{CT})\end{array}$ & $\begin{array}{l}3781(2765 \\
\mathrm{CT})\end{array}$ & $69.9 \mathrm{CT}$ \\
\hline Hoefler (2016) & $\begin{array}{l}\text { Mixed (MCI or } \\
\text { AD) }\end{array}$ & $\begin{array}{l}\text { CT } \\
\text { (computerized) }\end{array}$ & Not reported & $\begin{array}{l}\text { Attention/processing } \\
\text { speed } \\
\text { Global cognition } \\
\quad \text { (screening) } \\
\text { Verbal memory } \\
\text { Visual memory } \\
\text { Working memory and } \\
\text { learning } \\
\text { Mental health } \\
\text { ADL } \\
\text { Assessment of } \\
\text { dementia }\end{array}$ & 17 & 494 & $64-79.9$ \\
\hline $\begin{array}{l}\text { Hudes et al. } \\
\text { (2019) }\end{array}$ & Healthy OA & $\begin{array}{c}\text { CT (memory } \\
\text { strategy } \\
\text { training) }\end{array}$ & Not reported & $\begin{array}{l}\text { Memory self-efficacy } \\
\text { Memory strategy use } \\
\text { Memory-related affect } \\
\text { Perceived memory } \\
\quad \text { ability }\end{array}$ & 18 & 2895 & $50-99$ \\
\hline
\end{tabular}


Table 1 (continued)

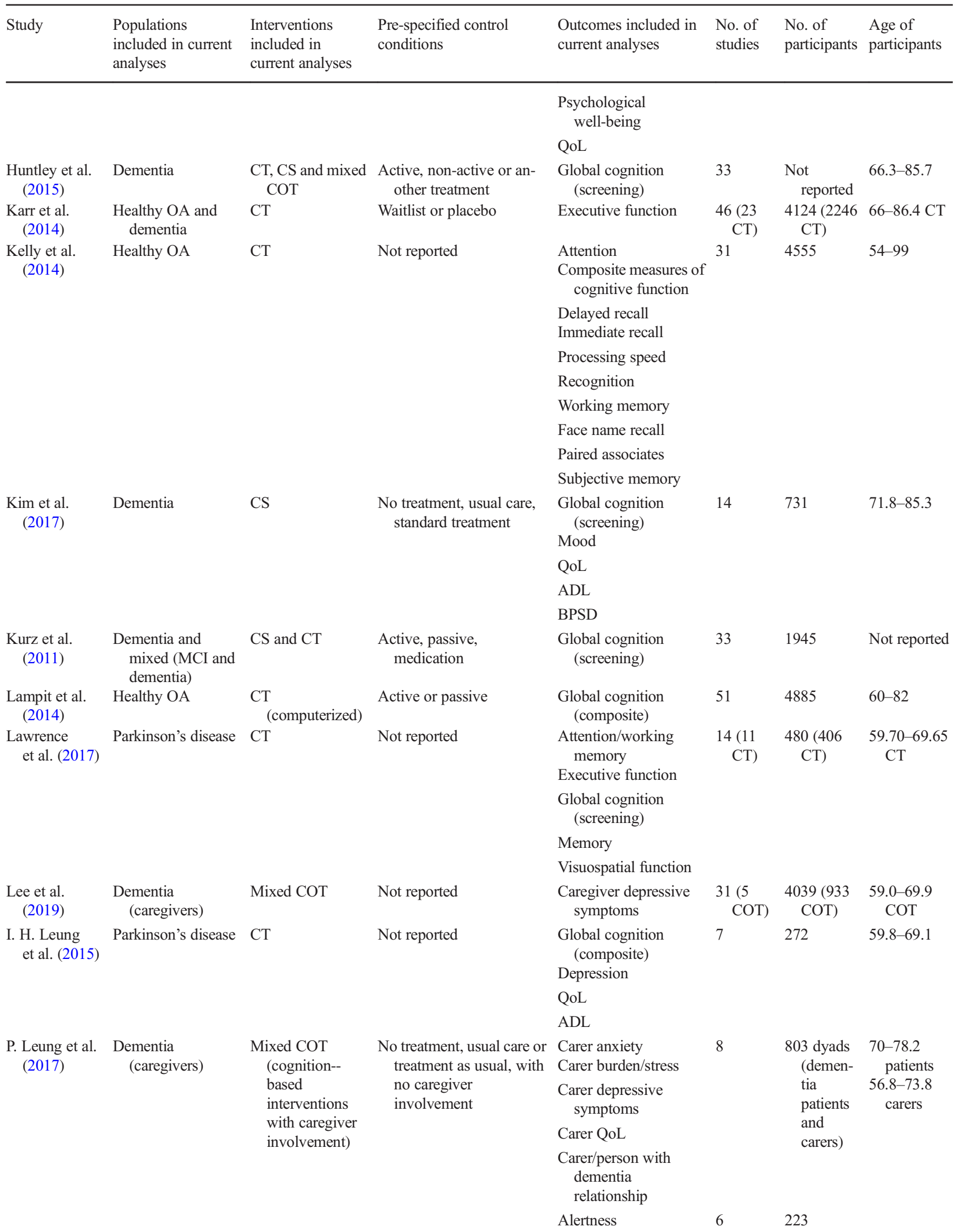


Table 1 (continued)

\begin{tabular}{|c|c|c|c|c|c|c|c|}
\hline Study & $\begin{array}{l}\text { Populations } \\
\text { included in current } \\
\text { analyses }\end{array}$ & $\begin{array}{l}\text { Interventions } \\
\text { included in } \\
\text { current analyses }\end{array}$ & $\begin{array}{l}\text { Pre-specified control } \\
\text { conditions }\end{array}$ & $\begin{array}{l}\text { Outcomes included in } \\
\text { current analyses }\end{array}$ & $\begin{array}{l}\text { No. of } \\
\text { studies }\end{array}$ & $\begin{array}{l}\text { No. of } \\
\text { participants }\end{array}$ & $\begin{array}{l}\text { Age of } \\
\text { participants }\end{array}$ \\
\hline $\begin{array}{l}\text { Loetscher and } \\
\text { Lincoln } \\
\text { (2013) }\end{array}$ & $\begin{array}{l}\text { Stroke (with } \\
\text { attentional } \\
\text { deficits) }\end{array}$ & $\begin{array}{l}\text { CT (focusing on } \\
\text { attentional } \\
\text { deficits) }\end{array}$ & $\begin{array}{l}\text { Alternative treatment } \\
\text { (computerised activities } \\
\text { with low attentional } \\
\text { demands or social } \\
\text { activities) or no } \\
\text { attentional intervention }\end{array}$ & $\begin{array}{l}\text { Divided attention } \\
\text { Selective attention } \\
\text { Sustained attention } \\
\text { Subjective attention } \\
\text { Mood } \\
\text { QoL } \\
\text { Functional abilities }\end{array}$ & & & $\begin{array}{l}49.5-70.2 \\
\text { treatment } \\
49.6-67.7 \\
\text { control }\end{array}$ \\
\hline $\begin{array}{l}\text { Martin et al. } \\
\quad(2011)\end{array}$ & $\begin{array}{l}\text { Healthy older adults } \\
\text { and MCI }\end{array}$ & $\begin{array}{l}\mathrm{CT} \text { (memory } \\
\text { training) }\end{array}$ & Active or no contact & $\begin{array}{l}\text { Delayed recall } \\
\text { Face-name delayed } \\
\quad \text { recall } \\
\text { Face-name immediate } \\
\quad \text { recall } \\
\text { Immediate recall } \\
\text { Paired associates } \\
\text { Short-term memory } \\
\text { Visuospatial memory }\end{array}$ & $\begin{array}{l}33 \\
\text { healt- } \\
\text { hy } \\
\text { OA } \\
3 \text { MCI }\end{array}$ & $\begin{array}{l}2229(2116 \\
\text { healthy } \\
\text { OA, } 113 \\
\text { MCI })\end{array}$ & 69.90 \\
\hline $\begin{array}{l}\text { Melby-Lervag } \\
\text { and Hulme } \\
\text { (2016) }\end{array}$ & Healthy OA & $\begin{array}{l}\text { CT (working } \\
\text { memory } \\
\text { training) }\end{array}$ & Not reported & Non-verbal reasoning & 17 & $\begin{array}{l}\text { Not } \\
\quad \text { reported }\end{array}$ & Not reported \\
\hline $\begin{array}{l}\text { Metternich } \\
\quad \text { et al. (2010) }\end{array}$ & $\begin{array}{l}\text { Healthy OA (with } \\
\text { subjective } \\
\text { memory } \\
\text { complaints or } \\
\text { desire to improve } \\
\text { memory } \\
\text { performance) }\end{array}$ & $\begin{array}{l}\mathrm{CT} \text { (memory } \\
\text { training) }\end{array}$ & Not reported & $\begin{array}{l}\text { Objective memory } \\
\text { Subjective memory } \\
\text { Depressive symptoms }\end{array}$ & 14 & 920 & $53-82$ \\
\hline $\begin{array}{l}\text { Mewborn } \\
\text { et al. (2017) }\end{array}$ & $\begin{array}{l}\text { Healthy OA and } \\
\text { MCI }\end{array}$ & $\mathrm{CT}$ & Active or passive & $\begin{array}{l}\text { Global cognition } \\
\text { (composite) }\end{array}$ & $\begin{array}{l}97(48 \\
\text { healt- } \\
\text { hy } \\
\text { O- } \\
\text { A,12 } \\
\text { MCI) }\end{array}$ & 8783 (total) & $63.75-85.13$ \\
\hline $\begin{array}{l}\text { Papp et al. } \\
\text { (2009) }\end{array}$ & Healthy OA & $\mathrm{CT}$ & Not reported & All outcomes & 10 & 4009 & Not reported \\
\hline $\begin{array}{l}\text { Pinquart and } \\
\text { Sörensen } \\
\text { (2001) }\end{array}$ & $\begin{array}{l}\text { Mixed (no } \\
\text { specification of } \\
\text { cognitive status) }\end{array}$ & $\mathrm{CT}$ & Untreated control group & $\begin{array}{l}\text { Clinican-rated } \\
\text { depression } \\
\text { Psychological } \\
\text { well-being } \\
\text { Self-rated depression }\end{array}$ & $\begin{array}{r}122(9 \\
\mathrm{CT})\end{array}$ & $\begin{array}{l}\text { Not } \\
\quad \text { reported }\end{array}$ & $55-87$ \\
\hline $\begin{array}{l}\text { Rogers et al. } \\
\quad(2018)\end{array}$ & Stroke & Mixed COT & $\begin{array}{l}\text { Treatment as usual, } \\
\text { placebo or waitlist } \\
\text { control }\end{array}$ & $\begin{array}{l}\text { Global cognition } \\
\text { (composite) }\end{array}$ & 22 & 1098 & $48-78$ \\
\hline $\begin{array}{l}\text { Shao et al. } \\
\text { (2015) }\end{array}$ & Healthy OA & $\begin{array}{l}\mathrm{CT} \\
\text { (computerised) }\end{array}$ & Not reported & $\begin{array}{l}\text { Executive function } \\
\text { Memory performance } \\
\text { Processing speed }\end{array}$ & 12 & 2008 & $66-82$ \\
\hline $\begin{array}{l}\text { Sherman et al. } \\
\text { (2017) }\end{array}$ & MCI & Mixed COT & Active or passive & $\begin{array}{l}\text { Global cognition } \\
\text { (composite) }\end{array}$ & 26 & $\begin{array}{l}876 \\
\quad \text { (training } \\
\text { groups) }\end{array}$ & $66-77$ \\
\hline $\begin{array}{l}\text { Smart et al. } \\
\text { (2017) }\end{array}$ & $\begin{array}{l}\text { Healthy OA (with } \\
\text { subjective } \\
\text { cognitive } \\
\text { decline) }\end{array}$ & CT & Not reported & $\begin{array}{l}\text { Global cognition } \\
\text { (composite) }\end{array}$ & 9 & 676 & $64.9-77.41$ \\
\hline $\begin{array}{l}\text { Song et al. } \\
\text { (2016) }\end{array}$ & $\begin{array}{l}\text { Dementia }(\mathrm{AD} \text { or } \\
\text { vascular } \\
\text { dementia) }\end{array}$ & CT & Not reported & $\begin{array}{l}\text { Global cognition } \\
\text { (composite) }\end{array}$ & 13 & 474 & $72.00-83.47$ \\
\hline
\end{tabular}


Table 1 (continued)

\begin{tabular}{|c|c|c|c|c|c|c|c|}
\hline Study & $\begin{array}{l}\text { Populations } \\
\text { included in current } \\
\text { analyses }\end{array}$ & $\begin{array}{l}\text { Interventions } \\
\text { included in } \\
\text { current analyses }\end{array}$ & $\begin{array}{l}\text { Pre-specified control } \\
\text { conditions }\end{array}$ & $\begin{array}{l}\text { Outcomes included in } \\
\text { current analyses }\end{array}$ & $\begin{array}{l}\text { No. of } \\
\text { studies }\end{array}$ & $\begin{array}{l}\text { No. of } \\
\text { participants }\end{array}$ & $\begin{array}{l}\text { Age of } \\
\text { participants }\end{array}$ \\
\hline $\begin{array}{l}\text { Tetlow and } \\
\text { Edwards } \\
(2017)\end{array}$ & Healthy OA & $\begin{array}{l}\text { CT (commercially } \\
\text { available) }\end{array}$ & Not reported & $\begin{array}{l}\text { Attention } \\
\text { Executive function } \\
\text { Memory } \\
\text { Processing speed } \\
\text { Reasoning } \\
\text { Verbal fluency } \\
\text { Visuospatial memory } \\
\text { Everyday function }\end{array}$ & 21 & 5201 & Not reported \\
\hline $\begin{array}{l}\text { Toril et al. } \\
\text { (2014) }\end{array}$ & Healthy OA & CT (videogames) & $\begin{array}{l}\text { No control group required } \\
\text { (only controlled trials } \\
\text { included in current } \\
\text { analysis) }\end{array}$ & $\begin{array}{l}\text { Global cognition } \\
\text { (composite) }\end{array}$ & 20 & 913 & Not reported \\
\hline $\begin{array}{l}\text { C. Wang et al. } \\
\text { (2014) }\end{array}$ & MCI & $\mathrm{CT}$ & Not reported & $\begin{array}{l}\text { Delayed memory } \\
\text { Executive function } \\
\text { Global cognition } \\
\quad \text { (screening) } \\
\text { Immediate memory } \\
\text { Working memory }\end{array}$ & $\begin{array}{r}18(11 \\
\mathrm{CT})\end{array}$ & $\begin{array}{l}1125(330 \\
\mathrm{CT})\end{array}$ & $\begin{array}{l}68-86 \\
\quad(68-78 \\
\quad \text { CT })\end{array}$ \\
\hline $\begin{array}{l}P \text {. Wang et al. } \\
\text { (2016) }\end{array}$ & Healthy OA & $\begin{array}{l}\text { CT (action } \\
\text { videogames) }\end{array}$ & Not reported & $\begin{array}{l}\text { Global cognition } \\
\text { (composite) }\end{array}$ & $\begin{array}{l}19(8 \\
\text { healt- } \\
\text { hy } \\
\text { OA) }\end{array}$ & $\begin{array}{c}636(255 \\
\text { healthy } \\
\text { OA) }\end{array}$ & $\begin{array}{l}\text { 65-74.8 } \\
\text { healthy } \\
\text { OA }\end{array}$ \\
\hline $\begin{array}{l}\text { Weicker et al. } \\
\text { (2016) }\end{array}$ & Healthy OA & $\begin{array}{l}\text { CT (working } \\
\text { memory } \\
\text { training) }\end{array}$ & Not reported & $\begin{array}{l}\text { Attention and } \\
\text { processing speed } \\
\text { Cognitive control and } \\
\text { executive function } \\
\text { Long-term memory } \\
\text { Reasoning and } \\
\text { intelligence } \\
\text { Working memory }\end{array}$ & $\begin{array}{l}103(23 \\
\text { healt- } \\
\text { hy } \\
\text { OA) }\end{array}$ & $\begin{array}{c}6113(978 \\
\text { healthy } \\
\text { OA) }\end{array}$ & Not reported \\
\hline $\begin{array}{c}\text { Virk et al. } \\
\text { (2015) }\end{array}$ & $\begin{array}{l}\text { Stroke (with } \\
\text { attentional } \\
\text { deficits) }\end{array}$ & $\begin{array}{l}\text { CT (focusing on } \\
\text { attentional } \\
\text { deficits) }\end{array}$ & Not reported & $\begin{array}{l}\text { Alternating attention } \\
\text { Divided attention } \\
\text { Inhibition } \\
\text { Selective attention } \\
\text { Sustained attention }\end{array}$ & $\begin{array}{l}12(6 \\
\text { strok- } \\
\text { e) }\end{array}$ & $\begin{array}{r}584(237 \\
\text { stroke })\end{array}$ & $\begin{array}{l}50.5-68.9 \\
\text { stroke }\end{array}$ \\
\hline $\begin{array}{l}\text { Woods et al. } \\
\text { (2012) }\end{array}$ & Dementia & $\mathrm{CS}$ & $\begin{array}{l}\text { No treatment, standard } \\
\text { treatment or placebo }\end{array}$ & $\begin{array}{l}\begin{array}{l}\text { Global cognition } \\
\text { (screening) }\end{array} \\
\text { Communication/social } \\
\quad \text { interaction } \\
\text { Mood (self-reported) } \\
\text { Mood (staff-reported) } \\
\text { Well-being and QoL } \\
\text { ADL } \\
\text { Caregiver depression } \\
\text { Carer stress/burden } \\
\text { Caregiver anxiety } \\
\text { Behaviour problem }\end{array}$ & 15 & 718 & $69.8-85.7$ \\
\hline $\begin{array}{l}\text { Yang et al. } \\
\text { (2018) }\end{array}$ & $\begin{array}{l}\text { Mixed (cognitive } \\
\text { decline, MCI, } \\
\text { dementia) }\end{array}$ & $\begin{array}{l}\text { Mixed COT } \\
\quad \text { (memory-- } \\
\text { focused } \\
\text { interventions) }\end{array}$ & Not reported & $\begin{array}{l}\text { Delayed recall } \\
\text { Global cognition } \\
\quad \text { (screening) } \\
\text { Immediate recall }\end{array}$ & 27 & 2177 & 75.80 \\
\hline
\end{tabular}


Table 1 (continued)

\begin{tabular}{|c|c|c|c|c|c|c|c|}
\hline Study & $\begin{array}{l}\text { Populations } \\
\text { included in current } \\
\text { analyses }\end{array}$ & $\begin{array}{l}\text { Interventions } \\
\text { included in } \\
\text { current analyses }\end{array}$ & $\begin{array}{l}\text { Pre-specified control } \\
\text { conditions }\end{array}$ & $\begin{array}{l}\text { Outcomes included in } \\
\text { current analyses }\end{array}$ & $\begin{array}{l}\text { No. of } \\
\text { studies }\end{array}$ & $\begin{array}{l}\text { No. of } \\
\text { participants }\end{array}$ & $\begin{array}{l}\text { Age of } \\
\text { participants }\end{array}$ \\
\hline & & & & \multicolumn{4}{|l|}{$\begin{array}{l}\text { Learning and memory } \\
\text { function }\end{array}$} \\
\hline & & & & \multicolumn{4}{|l|}{ Recognition } \\
\hline & & & & \multicolumn{4}{|l|}{$\begin{array}{l}\text { Subjective memory } \\
\text { performance }\end{array}$} \\
\hline & & & & \multicolumn{4}{|l|}{ Depression } \\
\hline
\end{tabular}

Note. Reviews may include interventions, populations and outcomes that are beyond the scope of this overview. In such cases, only the information of relevance for the current analyses is described. $\mathrm{AD}=$ Alzheimer's dementia; $\mathrm{CT}=$ cognitive training; $\mathrm{QoL}=$ quality of life; $\mathrm{ADL}=$ activities of daily living; $\mathrm{BPSD}=$ behavioural and psychological symptoms of dementia; $\mathrm{OA}=$ older adults; $\mathrm{MCI}=$ mild cognitive impairment; $\mathrm{COT}=$ cognition-oriented treatment, $\mathrm{CS}=$ cognitive stimulation

of these reviews included people with dementia living in long-term care facilities (Folkerts et al., 2017). Two reviews focused on PD (Lawrence, Gasson, Bucks, Troeung, \& Loftus, 2017; Leung et al., 2015) and four on individuals with stroke (das Nair, Cogger, Worthington, \& Lincoln, 2016; Loetscher \& Lincoln, 2013; Rogers, Foord, Stolwyk, Wong, \& Wilson, 2018; Virk, Williams, Brunsdon, Suh, \& Morrow, 2015). Five reviews were classified as a mixed population, three included individuals with MCI and dementia (Hoefler, 2016; Kurz, Leucht, \& Lautenschlager, 2011; Yang et al., 2018), one stated that they looked at participants without dementia (Floyd \& Scogin, 1997) and one did not specify cognitive status (Pinquart \& Sörensen, 2001). Four reviews synthesized pooled effects for more than one population. Two reviews reported meta-analytic results for healthy older adults and MCI (Martin, Clare, Altgassen, Cameron, \& Zehnder, 2011; Mewborn, Lindbergh, \& Stephen Miller, 2017). One review reported results for healthy older adults and dementia (Karr, Areshenkoff, Rast, \& GarciaBarrera, 2014), and one focused on individuals with MCI and dementia (Hill et al., 2017).

Interventions. In all, 34 reviews reported meta-analytic results for cognitive training (Table 1), covering a variety of cognitive training approaches, including computerized training (Gates et al., 2019; Gates, Vernooij, et al., 2019; Hill et al., 2017; Hoefler, 2016; Lampit, Hallock, \& Valenzuela, 2014; Shao et al., 2015; Tetlow \& Edwards, 2017), memory training (das Nair, Cogger, Worthington, \& Lincoln, 2016; Floyd \& Scogin, 1997; Gross et al., 2012; Hudes, Rich, Troyer, Yusupov, \& Vandermorris, 2019; Martin et al., 2011; Metternich et al., 2010), working memory training (Melby-Lervag \& Hulme, 2016; Weicker, Villringer, \& Thone-Otto, 2016) and videogames (Toril, Reales, \& Ballesteros, 2014; P. Wang et al., 2016). Three reviews focused on cognitive stimulation (C. Cooper et al., 2012; Kim et al., 2017; Woods et al.,
2012). No review reported meta-analytic results for cognitive rehabilitation. Three reviews reported meta-analytic results for both cognitive training and cognitive stimulation (Folkerts et al., 2017; Huntley et al., 2015; Kurz et al., 2011). Six were classified as mixed cognitionoriented treatments (Chandler et al., 2016; Lee et al., 2019; P. Leung et al., 2017; Rogers et al., 2018; Sherman et al., 2017; Yang et al., 2018), one of which focused specifically on cognition-oriented treatments with caregiver involvement (P. Leung et al., 2017).

\section{Quality Assessment}

The results from the AMSTAR ratings for each review are presented in Supplementary Material 5. Mean AMSTAR score was $7.95(\mathrm{SD}=3.33)$ out of 16 . The confidence ratings were moderate for $9(20 \%)$ of 46 reviews, low for 13 (28\%) and critically low for $24(52 \%)$. None of the reviews were classified as high confidence. The number of reviews that adhered to each of the AMSTAR items (i.e., receiving a "Partial Yes" or "Yes") is shown in Fig. 2. The best adherence was found for conducting a comprehensive literature search (Item 4), using the components of PICO when describing the research question and inclusion criteria (Item 1), reporting conflicts of interest (Item 16) and discussing the impact of heterogeneity on the results (Item 14). The items that most reviews failed to meet were reporting the sources of funding for included studies (Item 10), providing a justification for excluding individual studies (Item 7), and assessing the potential impact of risk of bias on the results (Item 12).

For the critical domains, 45 reviews $(98 \%)$ conducted a comprehensive literature search (Item 4). Twenty-seven reviews $(59 \%)$ used a satisfactory technique for assessing the risk of bias in individual studies (Item 9) and 23 reviews $(50 \%)$ accounted for risk of bias when interpreting the results (Item 13). Twenty-three reviews (50\%) adhered 
Table 2 Methods of Included Meta-Analyses

\begin{tabular}{|c|c|c|c|c|c|c|c|}
\hline Study & Protocol & $\begin{array}{l}\text { Includes only } \\
\text { RCTs }\end{array}$ & $\begin{array}{l}\text { Search } \\
\text { period } \\
\text { covered }\end{array}$ & Databases searched & $\begin{array}{l}\text { Type and method of } \\
\text { effect size }\end{array}$ & $\begin{array}{l}\text { Moderator } \\
\text { analyses } \\
\text { conducted }\end{array}$ & $\begin{array}{l}\text { Method of grading the } \\
\text { quality of the } \\
\text { evidence }\end{array}$ \\
\hline $\begin{array}{l}\text { Alves et al. } \\
\text { (2013) }\end{array}$ & No & Yes & $\begin{array}{l}\text { From } \\
\text { inception } \\
\text { to } \\
\text { Marc- } \\
\text { h } 2012\end{array}$ & $\begin{array}{l}\text { PubMed, PsychINFO, The } \\
\text { Cochrane Library, EMBASE, } \\
\text { metaRegister of Clinical Trials } \\
\text { (ISRCTN Register, NIH } \\
\text { ClinicalTrials.gov } \\
\text { Register - subset of random- } \\
\text { ized trial records), OVID all } \\
\text { EBM Reviews (Cochrane } \\
\text { DSR, ACP Journal Club, } \\
\text { DARE, CCTR, HTA, } \\
\text { NHSEED). }\end{array}$ & $\begin{array}{l}\text { MD and SMD } \\
\text { post-treatment } \\
\text { difference }\end{array}$ & No & Cochrane RoB tool \\
\hline $\begin{array}{l}\text { Bahar-Fuchs } \\
\text { et al. (2019) }\end{array}$ & Yes & Yes & $\begin{array}{l}\text { Until } \\
\text { Jul- } \\
\text { y } 2018\end{array}$ & ALOIS & $\begin{array}{l}\text { SMD pre-post dif- } \\
\text { ference }\end{array}$ & Yes & $\begin{array}{l}\text { Cochrane RoB tool, } \\
\text { GRADE }\end{array}$ \\
\hline $\begin{array}{l}\text { Bhome et al. } \\
\text { (2018) }\end{array}$ & Yes & Yes & $\begin{array}{l}\text { Until } \\
\text { August } \\
2017\end{array}$ & $\begin{array}{l}\text { PubMed, Web of Science, } \\
\text { Cochrane Systematic Reviews } \\
\text { Database, PsycINFO and } \\
\text { CIANHL }\end{array}$ & $\begin{array}{l}\text { Hedges' g } \\
\text { post-treatment } \\
\text { difference }\end{array}$ & Yes & $\begin{array}{l}\text { Critical Appraisal } \\
\text { Skills Programme }\end{array}$ \\
\hline $\begin{array}{l}\text { Chandler et al. } \\
\text { (2016) }\end{array}$ & No & No & $\begin{array}{l}\text { Until } \\
\text { October } \\
2015\end{array}$ & MEDLINE, PsycINFO & $\begin{array}{l}\text { Cohen's d pre-post } \\
\text { difference }\end{array}$ & Yes & Not reported \\
\hline $\begin{array}{l}\text { Chiu et al. } \\
\text { (2017) }\end{array}$ & No & Yes & $\begin{array}{l}\text { Until } \\
\text { Decemb- } \\
\text { er } 2016\end{array}$ & $\begin{array}{l}\text { Cochrane, PubMed, EMBASE, } \\
\text { MEDLINE, PsycINFO, and } \\
\text { CINAHL. }\end{array}$ & Hedges' g & Yes & Cochrane RoB tool \\
\hline $\begin{array}{l}\text { C. Cooper } \\
\text { et al. }(2012)\end{array}$ & No & Yes & $\begin{array}{l}\text { Unil } \\
\quad \text { January } \\
2011\end{array}$ & $\begin{array}{l}\text { PubMed, Web of Science, and } \\
\text { Cochrane systematic reviews }\end{array}$ & $\begin{array}{l}\text { SMD } \\
\text { post-treatment } \\
\text { difference }\end{array}$ & No & $\begin{array}{l}\text { Checklist from the } \\
\text { Critical Appraisal } \\
\text { Skills Program }\end{array}$ \\
\hline $\begin{array}{l}\text { das Nair et al. } \\
\text { (2016) }\end{array}$ & Yes & Yes & $\begin{array}{l}\text { Until } \\
\text { Ma- } \\
\text { y } 2016\end{array}$ & $\begin{array}{l}\text { Cochrane Stroke Group Trials } \\
\text { Register, Cochrane Central } \\
\text { Register of Controlled Trials, } \\
\text { MEDLINE (Ovid), EMBASE } \\
\text { (Ovid), CINAHL, AMED, } \\
\text { PsycINFO, ClinicalTrials.gov, } \\
\text { WHO International Clinical } \\
\text { Trials Registry Portal, NIHR } \\
\text { Clinical Research Network } \\
\text { Database, UK CRN Study } \\
\text { Portfolio, LILACS, CAB } \\
\text { Abstracts, REHABDATA, } \\
\text { Stroke Trials Registry, } \\
\text { ISRCTN Registry. }\end{array}$ & SMD & No & Cochrane RoB tool \\
\hline $\begin{array}{l}\text { Floyd and } \\
\text { Scogin } \\
(1997)\end{array}$ & No & No & 1970-1994 & $\begin{array}{l}\text { PsycLIT, Dissertation Abstracts } \\
\text { International }\end{array}$ & $\begin{array}{l}\text { Cohen's d, } \\
\text { post-treatment } \\
\text { difference }\end{array}$ & No & $\begin{array}{l}\text { "Scale developed by } \\
\text { Suydam (1968)" }\end{array}$ \\
\hline $\begin{array}{l}\text { Folkerts et al. } \\
\qquad(2017)\end{array}$ & No & $\begin{array}{l}\text { No } \\
\qquad \text { (meta-- } \\
\text { analysis } \\
\text { restricted to } \\
\text { RCTs) }\end{array}$ & $\begin{array}{l}\text { Until } \\
\text { Decemb- } \\
\text { er } 2015\end{array}$ & $\begin{array}{l}\text { PubMed, which is backed by the } \\
\text { MEDLINE database, and } \\
\text { CENTRAL (Cochrane Central } \\
\text { Register of Controlled Trials) }\end{array}$ & $\begin{array}{l}\text { SMD pre-post dif- } \\
\text { ference }\end{array}$ & Yes & Cochrane RoB tool \\
\hline $\begin{array}{l}\text { Gates, Rutjes, } \\
\text { et al. (2019) }\end{array}$ & Yes & Yes & $\begin{array}{l}\text { Until } \\
\text { Marc- } \\
\text { h } 2018\end{array}$ & ALOIS & $\begin{array}{l}\text { SMD pre-post dif- } \\
\text { ference }\end{array}$ & No & $\begin{array}{l}\text { Cochrane RoB tool, } \\
\text { GRADE }\end{array}$ \\
\hline $\begin{array}{l}\text { Gates, } \\
\text { Vernooij, } \\
\text { et al. (2019) }\end{array}$ & Yes & Yes & $\begin{array}{l}\text { Until } \\
\text { Ma- } \\
\text { y } 2018\end{array}$ & ALOIS & $\begin{array}{l}\text { SMD pre-post dif- } \\
\text { ference }\end{array}$ & No & $\begin{array}{l}\text { Cochrane RoB tool, } \\
\text { GRADE }\end{array}$ \\
\hline \multirow[t]{2}{*}{$\begin{array}{l}\text { Gross et al. } \\
\quad(2012)\end{array}$} & No & No & $\begin{array}{l}\text { Until } \\
\text { January } \\
2010\end{array}$ & PsychInfo, PsychLit, PubMed. & $\begin{array}{l}\text { SMD pre-post dif- } \\
\text { ference }\end{array}$ & Yes & Not reported \\
\hline & Yes & Yes & & & & No & \\
\hline
\end{tabular}


Table 2 (continued)

\begin{tabular}{|c|c|c|c|c|c|c|c|}
\hline Study & Protocol & $\begin{array}{l}\text { Includes only } \\
\text { RCTs }\end{array}$ & $\begin{array}{l}\text { Search } \\
\text { period } \\
\text { covered }\end{array}$ & Databases searched & $\begin{array}{l}\text { Type and method of } \\
\text { effect size }\end{array}$ & $\begin{array}{l}\text { Moderator } \\
\text { analyses } \\
\text { conducted }\end{array}$ & $\begin{array}{l}\text { Method of grading the } \\
\text { quality of the } \\
\text { evidence }\end{array}$ \\
\hline $\begin{array}{r}\text { Hill et al. } \\
\text { (2017) }\end{array}$ & & & $\begin{array}{l}\text { From } \\
\text { inception } \\
\text { to } \\
\text { Jul- } \\
\text { y } 2016\end{array}$ & $\begin{array}{l}\text { Medline, Embase, PsychINFO, } \\
\text { CINAHL, and CENTRAL. }\end{array}$ & $\begin{array}{l}\text { Hedges' g pre-post } \\
\text { difference }\end{array}$ & & $\begin{array}{l}\text { Cochrane RoB tool; } \\
\text { PEDro-P scale }\end{array}$ \\
\hline $\begin{array}{l}\text { Hindin and } \\
\text { Zelinski } \\
\text { (2012) }\end{array}$ & No & No & $\begin{array}{l}\text { Until } \\
\quad \text { January } \\
2011\end{array}$ & $\begin{array}{l}\text { PSYCINFO, Social Gerontology, } \\
\text { and MEDLINE. }\end{array}$ & $\begin{array}{l}\text { Cohen's d pre-post } \\
\text { difference }\end{array}$ & Yes & $\begin{array}{l}\text { A 5-point scale } \\
\text { adapted from items } \\
\text { used in Papp et al. } \\
\text { (2009) }\end{array}$ \\
\hline Hoefler (2016) & No & No & $1980-2015$ & Supersearch, Google Scholar. & $\begin{array}{l}\text { Cohen's d } \\
\text { post-treatment } \\
\text { difference }\end{array}$ & No & $\begin{array}{l}\text { 'Assessment of } \\
\text { methodological } \\
\text { quality (Lipsey \& } \\
\text { Wilson, 2009)' - } 10 \\
\text { item scale }\end{array}$ \\
\hline $\begin{array}{l}\text { Hudes et al. } \\
\text { (2019) }\end{array}$ & Yes & Yes & $\begin{array}{l}\text { Until } \\
\text { October } \\
2018\end{array}$ & $\begin{array}{l}\text { PsycINFO, MedLine, Cochrane } \\
\text { Central Register of Controlled } \\
\text { Trials, Cochrane Database of } \\
\text { Systematic Reviews. }\end{array}$ & Cohen's d & Yes & $\begin{array}{l}\text { Effective Public } \\
\text { Health Practice } \\
\text { Project tool }\end{array}$ \\
\hline $\begin{array}{l}\text { Huntley et al. } \\
\text { (2015) }\end{array}$ & No & Yes & $\begin{array}{l}\text { Until } \\
\text { Jun- } \\
\text { e } 2013\end{array}$ & $\begin{array}{l}\text { Web of Knowledge, Cochrane } \\
\text { Collaborative Central Register } \\
\text { of Controlled Trials, and } \\
\text { PubMed/Medline }\end{array}$ & $\begin{array}{l}\text { Hedges' g pre-post } \\
\text { difference }\end{array}$ & Yes & Cochrane RoB tool \\
\hline $\begin{array}{l}\text { Karr et al. } \\
\quad(2014)\end{array}$ & No & No & $\begin{array}{l}\text { Until } \\
\text { Jun- } \\
\text { e } 2013\end{array}$ & $\begin{array}{l}\text { PsychInfo, MedLine, CINAHL, } \\
\text { PsycArticles and Cochrane } \\
\text { Central Register of Controlled } \\
\text { Trials }\end{array}$ & $\begin{array}{l}\text { Cohen's, pre-post } \\
\text { difference }\end{array}$ & Yes & PEDro scale \\
\hline $\begin{array}{l}\text { Kelly et al. } \\
\text { (2014) }\end{array}$ & Yes & Yes & $\begin{array}{l}\text { From } 2002 \\
\quad \text { to } 2012\end{array}$ & $\begin{array}{l}\text { PubMed, Medline, the Cochrane } \\
\text { Library, and ClinicalTrials.gov, } \\
\text { Google Scholar. }\end{array}$ & $\begin{array}{l}\text { SMD pre-post dif- } \\
\text { ference }\end{array}$ & No & Cochrane RoB tool \\
\hline $\begin{array}{l}\text { Kim et al. } \\
\text { (2017) }\end{array}$ & No & Yes & $\begin{array}{r}1982 \text { to } \\
\text { April } \\
2015\end{array}$ & $\begin{array}{l}\text { PubMed, MEDLINE (1966 to } \\
\text { April 2015), Embase (1980 to } \\
\text { April 2015), PsychINFO, and } \\
\text { Cochrane Reviews Library }\end{array}$ & $\begin{array}{l}\text { Cohen's d pre-post } \\
\text { difference }\end{array}$ & No & Cochrane RoB tool \\
\hline $\begin{array}{l}\text { Kurz et al. } \\
\quad(2011)\end{array}$ & No & Yes & $\begin{array}{l}\text { Until } \\
\text { Decemb- } \\
\text { er } 2010\end{array}$ & $\begin{array}{l}\text { Medline, Science Citations Index } \\
\text { Expanded }\end{array}$ & $\begin{array}{l}\text { Hedges' g, } \\
\text { post-treatment } \\
\text { difference }\end{array}$ & No & Not reported \\
\hline $\begin{array}{l}\text { Lampit et al. } \\
\quad(2014)\end{array}$ & Yes & Yes & $\begin{array}{l}\text { From } \\
\text { inception } \\
\text { to } \\
\text { Jul- } \\
\text { y } 2014\end{array}$ & $\begin{array}{l}\text { Medline, Embase, and } \\
\text { PsychINFO }\end{array}$ & $\begin{array}{l}\text { Hedges' g pre-post } \\
\text { difference }\end{array}$ & Yes & $\begin{array}{l}\text { Cochrane RoB tool; } \\
\text { PEDro scale }\end{array}$ \\
\hline $\begin{array}{l}\text { Lawrence } \\
\quad \text { et al. (2017) }\end{array}$ & No & No & $\begin{array}{l}\text { From } \\
\text { inception } \\
\text { to } \\
\text { Ma- } \\
\text { y } 2016\end{array}$ & $\begin{array}{l}\text { MEDLINE, PubMed, Wiley } \\
\text { Online Library and gray } \\
\text { literature (e.g., OpenGrey, } \\
\text { NTIS). }\end{array}$ & $\begin{array}{l}\text { Hedges' g pre-post } \\
\text { difference }\end{array}$ & No & Cochrane RoB tool \\
\hline $\begin{array}{r}\text { Lee et al. } \\
(2019)\end{array}$ & No & Yes & $\begin{array}{l}\text { From } 2007 \\
\quad \text { to } 2017\end{array}$ & $\begin{array}{l}\text { MEDLINE (Ovid), CINAHL, } \\
\text { PsychInfo. }\end{array}$ & Cohen's d & No & Cochrane RoB tool \\
\hline $\begin{array}{l}\text { I. H. Leung } \\
\text { et al. (2015) }\end{array}$ & Yes & Yes & $\begin{array}{l}\text { From } \\
\text { inception } \\
\text { to } \\
\text { Novemb- } \\
\text { er } 2014\end{array}$ & $\begin{array}{l}\text { Medline (Ovid), Embase, } \\
\text { PsychInfo, CINAHL, and } \\
\text { CENTRAL }\end{array}$ & $\begin{array}{l}\text { Hedges' g pre-post } \\
\text { difference }\end{array}$ & No & $\begin{array}{l}\text { Cochrane RoB tool, } \\
\text { An adapted version } \\
\text { of the PEDro-P } \\
\text { scale }\end{array}$ \\
\hline $\begin{array}{l}\text { P. Leung et al. } \\
\text { (2017) }\end{array}$ & No & Yes & $\begin{array}{l}\text { Until } \\
\text { Decemb- } \\
\text { er } 2015\end{array}$ & $\begin{array}{l}\text { MEDLINE, Embase,Pubmed, } \\
\text { PsycINFO, Alois, Cumulative } \\
\text { Index of Nursing and Allied } \\
\text { Health Literature, Cochrane } \\
\text { Library }\end{array}$ & $\begin{array}{l}\text { Hedges' g pre-post } \\
\text { difference }\end{array}$ & No & Cochrane RoB tool \\
\hline
\end{tabular}


Table 2 (continued)

\begin{tabular}{|c|c|c|c|c|c|c|c|}
\hline Study & Protocol & $\begin{array}{l}\text { Includes only } \\
\text { RCTs }\end{array}$ & $\begin{array}{l}\text { Search } \\
\text { period } \\
\text { covered }\end{array}$ & Databases searched & $\begin{array}{l}\text { Type and method of } \\
\text { effect size }\end{array}$ & $\begin{array}{l}\text { Moderator } \\
\text { analyses } \\
\text { conducted }\end{array}$ & $\begin{array}{l}\text { Method of grading the } \\
\text { quality of the } \\
\text { evidence }\end{array}$ \\
\hline $\begin{array}{l}\text { Loetscher and } \\
\text { Lincoln } \\
\text { (2013) }\end{array}$ & Yes & Yes & $\begin{array}{l}\text { Until } \\
\text { October } \\
2012\end{array}$ & $\begin{array}{l}\text { CENTRAL, MEDLINE, } \\
\text { Embase, PsychInfo, CINAHL, } \\
\text { PsycBITE, REHABDATA, } \\
\text { ClinicalTrials.gov, Stroke } \\
\text { Trials Registry, Current } \\
\text { Controlled Trials }\end{array}$ & $\begin{array}{l}\text { MD and SMD, } \\
\text { pre-post differ- } \\
\text { ence }\end{array}$ & No & Cochrane RoB tool \\
\hline $\begin{array}{l}\text { Martin et al. } \\
\text { (2011) }\end{array}$ & Yes & Yes & $\begin{array}{l}\text { January } \\
\text { 1970to } \\
\text { Septemb- } \\
\text { er } 2007\end{array}$ & $\begin{array}{l}\text { CENTRAL, MEDLINE, } \\
\text { Embase, PsychInfo, CINAHL, } \\
\text { SIGLE, LILACS }\end{array}$ & $\begin{array}{l}\text { MD and SMD, } \\
\text { pre-post differ- } \\
\text { ence }\end{array}$ & No & $\begin{array}{l}\text { Methodological } \\
\text { quality of } \\
\text { randomisation } \\
\text { assessed as } \\
\text { described in } \\
\text { Cochrane } \\
\text { Handbook }\end{array}$ \\
\hline $\begin{array}{l}\text { Melby-Lervag } \\
\text { and Hulme } \\
\text { (2016) }\end{array}$ & No & No & $\begin{array}{l}\text { Not } \\
\quad \text { reported }\end{array}$ & $\begin{array}{l}\text { PsychInfo, PsycArticles, Medline } \\
\text { and Google Scholar and ERIC }\end{array}$ & $\begin{array}{l}\text { Hedges' g pre-post } \\
\text { difference }\end{array}$ & No & Not reported \\
\hline $\begin{array}{l}\text { Metternich } \\
\text { et al. (2010) }\end{array}$ & No & Yes & $\begin{array}{l}\text { Not } \\
\quad \text { reported }\end{array}$ & MedLine and PsycInfo & $\begin{array}{l}\text { Hedges' g pre-post } \\
\text { difference }\end{array}$ & No & In-house developed \\
\hline $\begin{array}{l}\text { Mewborn } \\
\text { et al. (2017) }\end{array}$ & Yes & Yes & $\begin{array}{l}\text { Until } \\
\text { Ma- } \\
\text { y } 2016\end{array}$ & $\begin{array}{l}\text { EBSCOhost onlinedatabases } \\
\text { (Academic Search Complete, } \\
\text { AgeLine,MEDLINE, } \\
\text { PsycARTICLES, Psychology } \\
\text { and BehavioralSciences } \\
\text { Collection, and PsycINFO). }\end{array}$ & $\begin{array}{l}\text { Hedges' g pre-post } \\
\text { difference }\end{array}$ & Yes & Cochrane RoB tool \\
\hline $\begin{array}{l}\text { Papp et al. } \\
\text { (2009) }\end{array}$ & No & Yes & $\begin{array}{l}\text { From } 1992 \\
\text { to } \\
\text { Decemb- } \\
\text { er } 2007\end{array}$ & $\begin{array}{l}\text { MEDLINE, Scopus, } \\
\text { TheCochrane Collaboration, } \\
\text { Dissertation Abstract } \\
\text { International, and PsycINFO. } \\
\text { Registers - Current Controlled } \\
\text { Trials and Clinicaltrials.gov. }\end{array}$ & $\begin{array}{l}\text { SMD } \\
\text { post-treatment } \\
\text { difference }\end{array}$ & No & $\begin{array}{l}\text { Combination of items } \\
\text { from a modified } \\
\text { version of the Scale } \\
\text { to Assess Scientific } \\
\text { Quality of } \\
\text { Investigations and } \\
\text { Jadad }\end{array}$ \\
\hline $\begin{array}{l}\text { Pinquart and } \\
\text { Sörensen } \\
(2001)\end{array}$ & No & Not reported & $\begin{array}{l}\text { Not } \\
\text { reported }\end{array}$ & $\begin{array}{l}\text { MEDLINE, PsycINFO, } \\
\text { PSYNDEX }\end{array}$ & $\begin{array}{l}\text { Hedges' g } \\
\text { post-treatment } \\
\text { difference }\end{array}$ & Yes & $\begin{array}{l}\text { Quality of research } \\
\text { report coded by } \\
\text { scale of } 1 \text { to } 3\end{array}$ \\
\hline $\begin{array}{l}\text { Rogers et al. } \\
\text { (2018) }\end{array}$ & Yes & Yes & $\begin{array}{l}\text { Until } \\
\text { Decemb- } \\
\text { er } 2017\end{array}$ & $\begin{array}{l}\text { AMED, CINAHL, Cochrane } \\
\text { Library, EMBASE, } \\
\text { MEDLINE, PsycEXTRA, } \\
\text { PsycINFO, Science Direct, } \\
\text { Scopus. }\end{array}$ & $\begin{array}{l}\text { Hedges' g } \\
\text { post-treatment } \\
\text { difference }\end{array}$ & Yes & PEDro \\
\hline $\begin{array}{l}\text { Shao et al. } \\
\text { (2015) }\end{array}$ & No & Yes & $\begin{array}{l}\text { From } 2000 \\
\quad \text { to } \\
\text { October } \\
2014\end{array}$ & $\begin{array}{l}\text { Pubmed, EMBASE, Cochrane } \\
\text { Library, China Knowledge } \\
\text { Resource Integrated Database, } \\
\text { Wan Fang Data and Weipu } \\
\text { Database for Chinese } \\
\text { Technical Periodicals. }\end{array}$ & SMD & Yes & Cochrane RoB tool \\
\hline $\begin{array}{l}\text { Sherman et al. } \\
\text { (2017) }\end{array}$ & No & Yes & $\begin{array}{l}\text { From } \\
\text { January } \\
1995 \text { to } \\
\text { Jun- } \\
\text { e } 2017\end{array}$ & $\begin{array}{l}\text { MEDLINE-R, PubMed, } \\
\text { Healthstar, Global Health, } \\
\text { PSYCH-INFO, Health and } \\
\text { Psychological Instruments. }\end{array}$ & $\begin{array}{l}\text { Hedges' g } \\
\text { post-treatment } \\
\text { difference }\end{array}$ & Yes & $\begin{array}{l}\text { NIH Quality of } \\
\text { Assessment of } \\
\text { Controlled } \\
\text { Intervention } \\
\text { Studies Scale }\end{array}$ \\
\hline $\begin{array}{l}\text { Smart et al. } \\
\quad(2017)\end{array}$ & No & No & $\begin{array}{l}\text { Until } \\
\quad \text { Novemb- } \\
\text { er } 2015\end{array}$ & $\begin{array}{l}\text { CINAHL Complete,Cochrane } \\
\text { Central Register of Controlled } \\
\text { Trials, MEDLINEwith Full } \\
\text { Text, PsycINFO, and } \\
\text { PsycARTICLES }\end{array}$ & $\begin{array}{l}\text { Bayesian pre-post } \\
\text { difference }\end{array}$ & No & PEDro \\
\hline $\begin{array}{l}\text { Song et al. } \\
\text { (2016) }\end{array}$ & No & Yes & $\begin{array}{l}\text { From } \\
\text { January } \\
2001 \text { to }\end{array}$ & $\begin{array}{l}\text { Cochrane Database, } \\
\text { EBSCO(CINAHL), PubMed, } \\
\text { ProQuest, and ScienceDirect }\end{array}$ & Not reported & No & Jadad \\
\hline
\end{tabular}


Table 2 (continued)

\begin{tabular}{|c|c|c|c|c|c|c|c|}
\hline Study & Protocol & $\begin{array}{l}\text { Includes only } \\
\text { RCTs }\end{array}$ & $\begin{array}{l}\text { Search } \\
\text { period } \\
\text { covered }\end{array}$ & Databases searched & $\begin{array}{l}\text { Type and method of } \\
\text { effect size }\end{array}$ & $\begin{array}{l}\text { Moderator } \\
\text { analyses } \\
\text { conducted }\end{array}$ & $\begin{array}{l}\text { Method of grading the } \\
\text { quality of the } \\
\text { evidence }\end{array}$ \\
\hline & & & $\begin{array}{l}\text { April } \\
2015\end{array}$ & & & & \\
\hline $\begin{array}{l}\text { Tetlow and } \\
\text { Edwards } \\
(2017)\end{array}$ & Yes & Yes & $\begin{array}{l}\text { Until } 28 \\
\text { Jul- } \\
\text { y } 2017\end{array}$ & Google, PubMed, PsychINFO & $\begin{array}{l}\text { Cohen's d } \\
\text { post-treatment } \\
\text { difference }\end{array}$ & Yes & PEDro \\
\hline $\begin{array}{l}\text { Toril et al. } \\
\text { (2014) }\end{array}$ & No & No & $\begin{array}{l}\text { From } 1986 \\
\quad \text { to } 2013\end{array}$ & $\begin{array}{l}\text { MEDLINE,PsychInfo, and } \\
\text { Google Scholar }\end{array}$ & $\begin{array}{l}\text { Cohen's d pre-post } \\
\text { difference }\end{array}$ & Yes & Not reported \\
\hline $\begin{array}{l}\text { C. Wang et al. } \\
\text { (2014) }\end{array}$ & No & Yes & $\begin{array}{l}\text { From } \\
\quad \text { January } \\
1990 \\
\text { toJanuary } \\
2014\end{array}$ & $\begin{array}{l}\text { MEDLINE, EMBASE, the } \\
\text { Cochranelibrary, and BIOSIS }\end{array}$ & MD and SMD & Yes & $\begin{array}{l}\text { Jadad and Grade } \\
\text { profiler } 3.6\end{array}$ \\
\hline $\begin{array}{l}\text { P. Wang et al. } \\
\text { (2016) }\end{array}$ & No & No & $\begin{array}{l}\text { From } \\
\text { January } \\
1986 \text { to } \\
\text { Jul- } \\
\text { y } 2015\end{array}$ & $\begin{array}{l}\text { Web of Sciences, } \\
\text { PubMed,EBSCO, PsycNET } \\
\text { (PsycINFO, PsycARTICLES) }\end{array}$ & $\begin{array}{l}\text { Cohen's d pre-post } \\
\text { difference }\end{array}$ & Yes & $\begin{array}{l}\text { 10-item scale was } \\
\text { developed }\end{array}$ \\
\hline $\begin{array}{l}\text { Weicker et al. } \\
\text { (2016) }\end{array}$ & No & No & $\begin{array}{l}\text { Until } \\
\quad \text { January } \\
2015\end{array}$ & $\begin{array}{l}\text { PubMed, OvidSP } \\
\text { (PsycINFO/PSYNDEX/Medl- } \\
\text { ine) }\end{array}$ & $\begin{array}{l}\text { Hedges' g } \\
\text { post-treatment } \\
\text { difference }\end{array}$ & Yes & Not reported \\
\hline $\begin{array}{l}\text { Virk et al. } \\
\text { (2015) }\end{array}$ & No & Yes & $\begin{array}{l}\text { From } \\
\text { inception } \\
\text { to } \\
\text { Augus- } \\
\text { t2014 }\end{array}$ & $\begin{array}{l}\text { MEDLINE, EMBASE, } \\
\text { PsycINFO andthe Cochrane } \\
\text { Central Register of Controlled } \\
\text { Trials(CENTRAL) }\end{array}$ & Hedges'g & Yes & Cochrane RoB tool \\
\hline $\begin{array}{l}\text { Woods et al. } \\
\text { (2012) }\end{array}$ & Yes & Yes & $\begin{array}{l}\text { Until } \\
\text { Decemb- } \\
\text { er } 2011\end{array}$ & $\begin{array}{l}\text { ALOIS which includes - } \\
\text { MEDLINE, EMBASE, } \\
\text { CINAHL, PsycINFO } \\
\text { andLILACS; CENTRAL; trial } \\
\text { registers, }\end{array}$ & $\begin{array}{l}\text { MD and SMD } \\
\text { pre-post differ- } \\
\text { ence }\end{array}$ & No & Cochrane RoB tool \\
\hline $\begin{array}{l}\text { Yang et al. } \\
\quad \text { (2018) }\end{array}$ & No & Yes & $\begin{array}{l}\text { Until } \\
\text { Ma- } \\
\text { y } 2017\end{array}$ & $\begin{array}{l}\text { PubMed, Cochrane Library, } \\
\text { Ovid-Medline, CINHAL, } \\
\text { PsycINFO, Ageline, Embase, } \\
\text { Google Scholar. }\end{array}$ & $\begin{array}{l}\text { Hedges' g pre-post } \\
\text { difference }\end{array}$ & Yes & Cochrane RoB tool \\
\hline
\end{tabular}

Note . $\mathrm{RCT}=$ randomized controlled trial; $\mathrm{MD}=$ mean difference; $\mathrm{SMD}=$ standardized mean difference; $\mathrm{RoB}=$ risk of bias; $\mathrm{PEDro}=\mathrm{Physiotherapy}$ Evidence Database Rating Scale

to the item of using appropriate methods for statistical combination of the results (Item 11) and 28 reviews $(61 \%)$ investigated publication bias (Item 15).

There was a significant positive association between AMSTAR score and year of publication (Spearman's rho = $0.30, p=0.04)$. However, when restricting the analysis to reviews that were published during the last ten years, thus excluding three older reviews (Floyd \& Scogin, 1997; Papp, Walsh, \& Snyder, 2009; Pinquart \& Sörensen, 2001), there was no significant association (Spearman's rho $=0.18, p=$ 0.24). Reviews that had a protocol had a higher AMSTAR score [mean 10.31 (SD=2.90) vs. mean $6.68(\mathrm{SD}=2.85)$, $\mathrm{t}(44)=4.09, p<0.001]$, as did reviews that only included RCTs [mean $8.80(\mathrm{SD}=3.19)$ vs. mean $6.00(\mathrm{SD}=2.88)$, $\mathrm{t}(44)=2.81, p<0.01]$.

\section{Cognitive Outcomes}

A total of 39 out of 46 reviews reported at least one pooled effect estimate for objective cognitive outcomes, providing a total of 47 effect estimates (Fig. 3).

Nineteen reviews investigated the effects of cognitive training on objective cognitive outcomes in healthy older adults. The mean effect estimate was 0.32 (0.05 to 0.59). Evidence from the two most comprehensive reviews with moderate confidence ratings showed a small and significant effect in favour of cognitive training on overall cognitive functioning. Lampit et al. (2014) synthesized the results of 51 trials of computerized cognitive training and reported an effect size of Hedges' $g=0.22$ (0.15 to 0.29) and Mewborn et al. (2017) pooled the results of 48 trials with an overall effect size of Hedges' $g=0.31$ (0.24 to 0.39). In all, 15 of 19 reviews showed a significant intervention effect on objective cognitive 
Table 3 Number of Identified Reviews Reporting Meta-Analytic Results for the Different Types of Interventions, Populations and Outcomes.

\begin{tabular}{|c|c|c|c|c|}
\hline & $\mathbf{C T}$ & CR & CS & COT \\
\hline Healthy OA & 20 & $\mathbf{0}$ & $\mathbf{0}$ & $\mathbf{0}$ \\
\hline $\begin{array}{l}\text { Objective cognition } \\
\text { Subjective cognition } \\
\text { Psychosocial } \\
\text { Functional } \\
\text { Caregiver } \\
\text { Clinical } \\
\text { BPSD } \\
\end{array}$ & $\begin{array}{c}19 \\
4 \\
3 \\
1\end{array}$ & & & \\
\hline MCI & 5 & $\mathbf{0}$ & $\mathbf{0}$ & 2 \\
\hline $\begin{array}{l}\text { Objective cognition } \\
\text { Subjective cognition } \\
\text { Psychosocial } \\
\text { Functional } \\
\text { Caregiver } \\
\text { Clinical } \\
\text { BPSD } \\
\end{array}$ & $\begin{array}{l}2 \\
2\end{array}$ & & & $\begin{array}{l}1 \\
1 \\
1 \\
1\end{array}$ \\
\hline Dementia & 7 & $\mathbf{0}$ & 6 & 3 \\
\hline $\begin{array}{l}\text { Objective cognition } \\
\text { Subjective cognition } \\
\text { Psychosocial } \\
\text { Functional } \\
\text { Caregiver } \\
\text { Clinical } \\
\text { BPSD } \\
\end{array}$ & $\begin{array}{l}7 \\
1 \\
2 \\
2 \\
1 \\
1 \\
1\end{array}$ & & $\begin{array}{l}4 \\
2 \\
1\end{array}$ & 1 \\
\hline Parkinson & 2 & $\mathbf{0}$ & $\mathbf{0}$ & $\mathbf{0}$ \\
\hline $\begin{array}{l}\text { Objective cognition } \\
\text { Subjective cognition } \\
\text { Psychosocial } \\
\text { Functional } \\
\text { Caregiver } \\
\text { Clinical } \\
\text { BPSD } \\
\end{array}$ & $\begin{array}{l}1 \\
1\end{array}$ & & & \\
\hline Stroke & 3 & $\mathbf{0}$ & $\mathbf{0}$ & 1 \\
\hline $\begin{array}{l}\text { Objective cognition } \\
\text { Subjective cognition } \\
\text { Psychosocial } \\
\text { Functional } \\
\text { Caregiver } \\
\text { Clinical } \\
\text { BPSD }\end{array}$ & $\begin{array}{l}3 \\
2 \\
2 \\
2\end{array}$ & & & 1 \\
\hline Mixed & 4 & $\mathbf{0}$ & $\mathbf{0}$ & 1 \\
\hline $\begin{array}{l}\text { Objective cognition } \\
\text { Subjective cognition } \\
\text { Psychosocial } \\
\text { Functional } \\
\text { Caregiver } \\
\text { Clinical } \\
\text { BPSD } \\
\end{array}$ & $\begin{array}{l}2 \\
1 \\
3 \\
1\end{array}$ & & & $\begin{array}{l}1 \\
1 \\
1\end{array}$ \\
\hline
\end{tabular}

Note. The number of identified reviews is colour coded: Grey $=$ no reviews; Yellow $=1-2$ reviews; Orange $=3-5$ reviews; Red $=>5$ reviews. $\mathrm{CT}=$ cognitive training; $\mathrm{CR}=$ cognitive rehabilitation; $\mathrm{CS}=$ cognitive stimulation; $\mathrm{COT}=$ mixed cognition-oriented treatment; $\mathrm{OA}=$ older adults; $\mathrm{BPSD}=$ behavioural and psychological symptoms of dementia

outcomes (Fig. 3). In contrast, one review of computerized cognitive training with low confidence AMSTAR rating showed a non-significant effect with large imprecision (Gates, Rutjes, et al., 2019) and three additional reviews with AMSTAR ratings in the critically low range showed no significant effect (Martin et al., 2011; Melby-Lervag \& Hulme, 2016; Tetlow \& Edwards, 2017).

Five reviews reported meta-analytic results for cognitive training for MCI. The mean effect size estimate was 0.40 (0.03 to 0.78). Evidence from the two most recent and comprehensive reviews with moderate confidence AMSTAR ratings showed a small and significant interventional effect (Hill et al., 2017; Mewborn et al., 2017) with Hedges' $g=0.35$ ( 0.20 to 0.50$)$ and 0.34 (0.21 to 0.47$)$, respectively.

For dementia, five reviews reported meta-analytic results for cognitive stimulation. The mean effect size estimate was 0.36 ( 0.15 to 0.57$)$. Evidence from the most recent review with a moderate confidence AMSTAR rating by Huntley et al. (2015) showed a small and significant effect of cognitive stimulation on global cognitive screening measures, Hedges' $g=0.35$ (0.11 to 0.58 ). Seven reviews reported meta-analytic results for cognitive training in people with dementia. The mean effect size estimate was $0.38(-0.04$ to 0.80$)$ and the results were heterogeneous (Fig. 3). The most recent and comprehensive review with a moderate confidence rating by Bahar-Fuchs et al. (2019) showed a small and significant effect on cognitive outcomes, Hedges' $g=0.42$ (0.23 to 0.61 ).

Two reviews assessed the effects of cognitive training in PD (Lawrence et al., 2017; Leung et al., 2015), both showed a small and significant interventional effect on cognitive outcomes.

Three reviews reported meta-analytic results for cognitive training for stroke (das Nair et al., 2016; Loetscher \& Lincoln, 2013; Virk et al., 2015). The mean effect estimate was 0.23 $(-0.19$ to 0.65$)$. The most recent review with a moderate confidence AMSTAR rating (Virk et al., 2015) focused specifically on treatment of attentional deficits, showing no significant effect, Hedges' $g=0.18$ ( -0.24 to 0.60$)$. In contrast, a moderate quality review on mixed cognition-oriented treatments for stroke revealed a small and significant effect on overall cognitive outcomes, Hedges' $g=0.48$ (0.36 to 0.60: Rogers et al., 2018). The mean effect estimates were robust to sensitivity analyses, in which reviews with a critically low confidence rating were excluded from the analysis (see Supplementary Material 6).

\section{Subjective Cognitive Outcomes}

Ten out of 46 reviews reported meta-analytic results on subjective cognitive outcomes (Fig. 4). Four reviews evaluated the effects of cognitive training on subjective cognitive complaints in healthy older adults, with confidence ratings in the low (Bhome et al., 2018; Hudes et al., 2019) or critically low range (Kelly et al., 2014; Metternich et al., 2010). The mean effect estimate was 0.29 (0.04 to 0.54$)$. The two most recent 


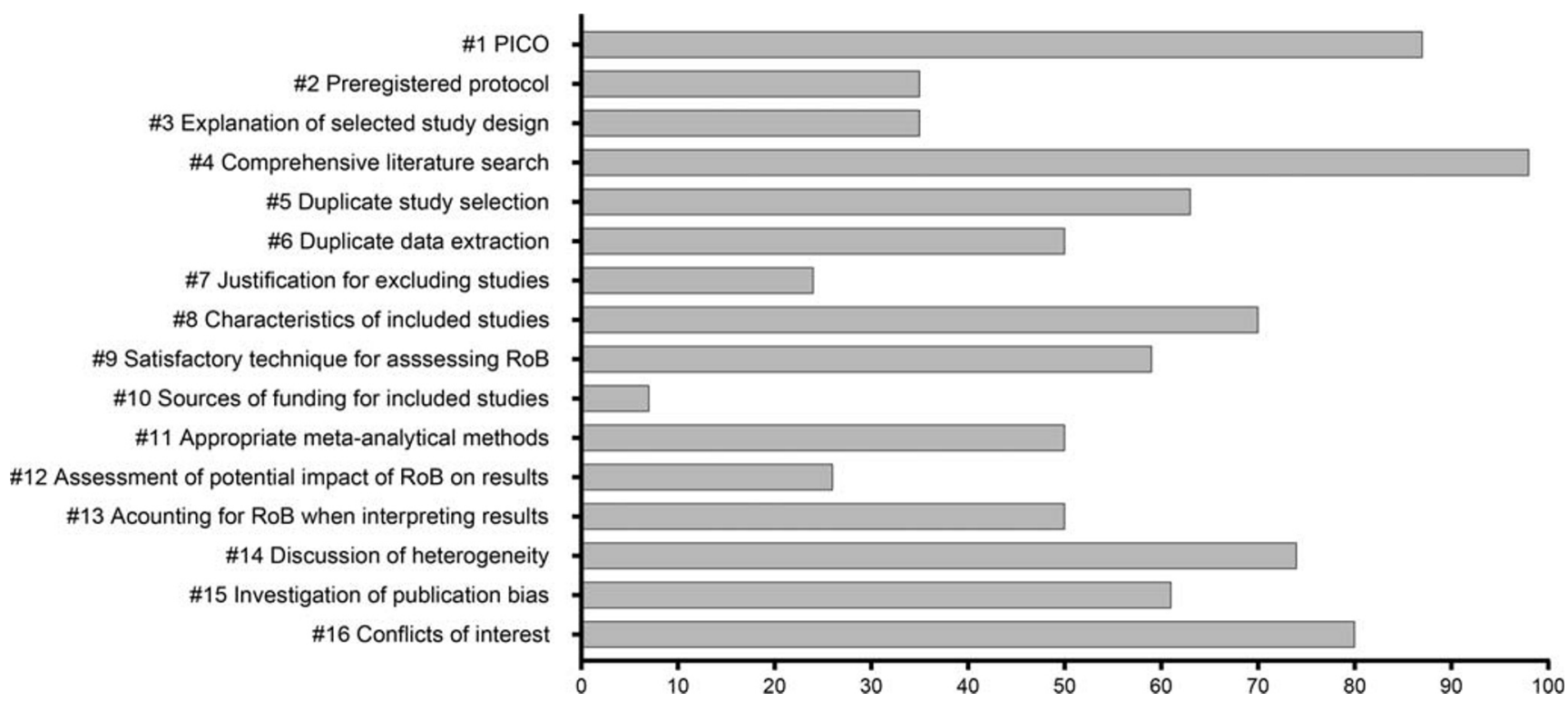

Fig. 2 Number of meta-analyses (percent) that adhered to the AMSTAR items AMSTAR $=$ a measurement tool to assess systematic reviews; $\mathrm{PICO}=$ population, intervention, comparator group, outcome; RoB $=$ risk of bias.

reviews with low confidence ratings showed results as follows. Bhome et al. (2018) found no significant effect of cognitive training for individuals with subjective cognitive complaints on metacognition, Hedges' $g=0.06$ ( -0.12 to 0.24$)$. In contrast, Hudes et al. (2019) investigated the effects of memory-strategy training on a number of meta-memory outcomes, showing a moderate and significant effect, Hedges' $g$ $=0.66(0.23$ to 1.08$)$.

For MCI, the review by Chandler et al. (2016) rated as critically low, demonstrated a small and significant effect of mixed cognition-oriented treatments on metacognitive outcomes. No significant effect of cognitive training on subjective cognition was found for dementia (Bahar-Fuchs et al., 2019). For stroke, one review demonstrated a small and significant improvement favouring cognitive training (das Nair et al., 2016), whereas another review demonstrated a nonsignificant effect with large imprecision (Loetscher \& Lincoln, 2013).

\section{Psychosocial Outcomes}

Eighteen out of 46 reviews assessed psychosocial outcomes, providing 19 effect estimates (Fig. 4). Three reviews assessed the effects of cognitive training on psychosocial outcomes for healthy older adults. The mean effect estimate was $0.23(-0.11$ to 0.57 ). The two most recent reviews, both rated as a low confidence, showed a small and significant effect on psychosocial outcomes with Hedges' $g=0.25$ ( 0.05 to 0.45 ) and 0.42 (0.09 to 0.74 ), respectively (Bhome et al., 2018; Hudes et al., 2019). For MCI, evidence from the most comprehensive, moderate quality review by Hill et al. (2017), showed a moderate significant effect of computerized cognitive training on psychosocial functioning, whereas a non-significant effect with large imprecision was reported in a more recent review rated as low confidence (Gates, Vernooij, et al., 2019). No significant benefits of cognitive training on psychosocial outcomes was found for dementia (Bahar-Fuchs et al., 2019; Hill et al., 2017), PD (I. H. Leung et al., 2015) or stroke (das Nair et al., 2016; Loetscher \& Lincoln, 2013).

Four reviews assessed the effects of cognitive stimulation on psychosocial outcomes for dementia. The mean effect size estimate was 0.26 ( -0.11 to 0.64$)$. Evidence from one moderate quality review by Woods et al. (2012) showed a borderline significant effect of Hedges' $g=0.27$ (-0.004 to 0.55 ) on the psychosocial composite outcome (Fig. 4). Authors reported significant benefits of cognitive stimulation on quality of life and staff ratings of communication and social interaction, but not for self- or staff-reported mood (Woods et al., 2012).

Four reviews assessed psychosocial outcomes in mixed populations (Floyd \& Scogin, 1997; Hoefler, 2016; Pinquart \& Sörensen, 2001; Yang et al., 2018), the most recent one was rated as low confidence, showing a moderate effect of mixed cognition-oriented treatments on depressive symptoms for individuals with MCI and dementia (Yang et al., 2018).

\section{Functional Outcomes}

Eleven out of 46 reviews assessed functional outcomes (Fig. 4), providing 12 effect size estimates, one in healthy older adults (Tetlow \& Edwards, 2017) three in MCI (mixed cognitionoriented treatments, Chandler et al., 2016; cognitive training, Gates, Vernooij, et al., 2019; Hill et al., 2017), four in dementia (cognitive training, Bahar-Fuchs et al., 2019; Hill et al., 2017; cognitive stimulation, Kim et al., 2017; Woods et al., 2012), one 


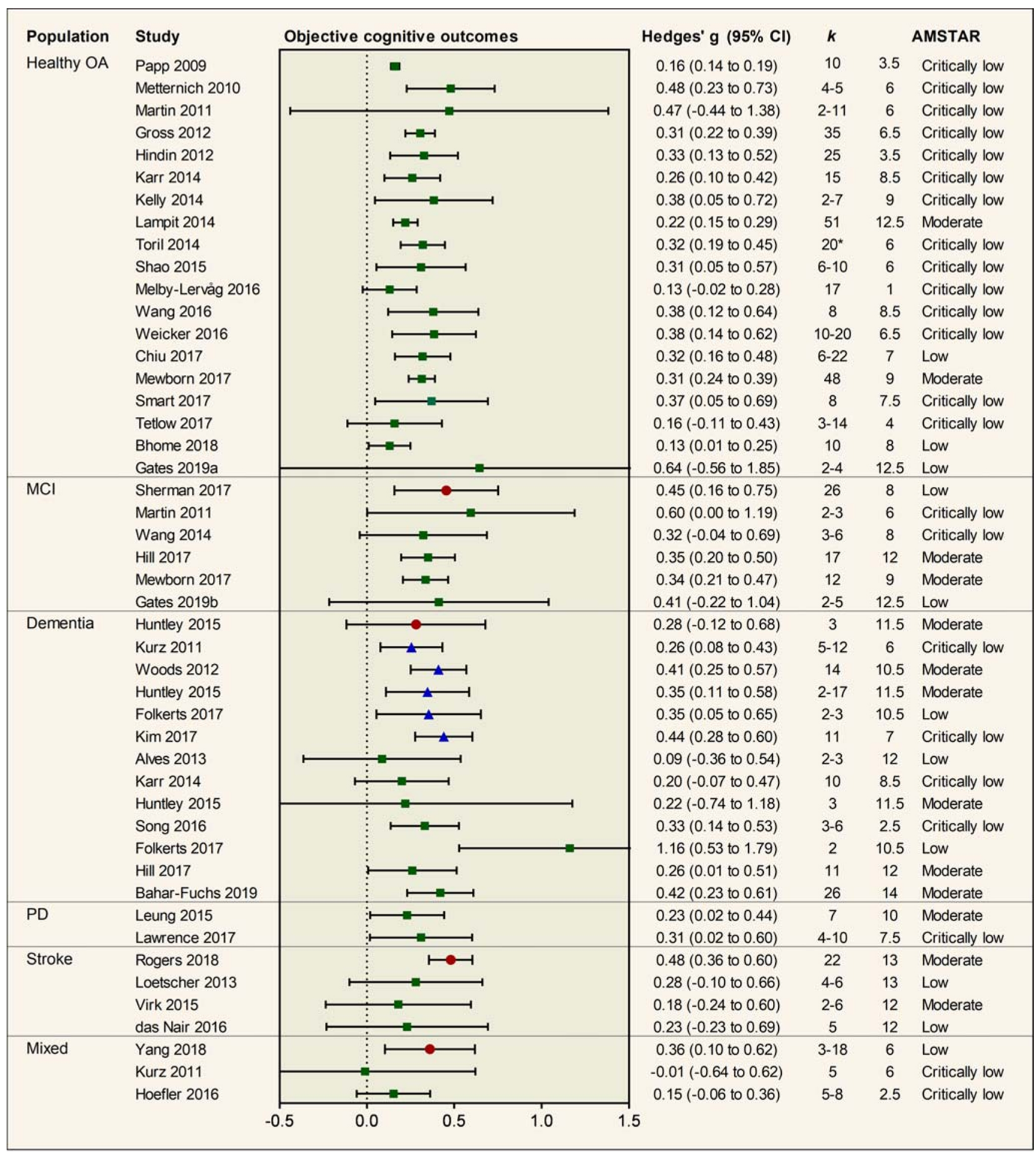

Fig. 3 Pooled results of meta-analyses investigating objective cognitive outcomes of cognition-oriented treatments (COT) in older adults. Positive values represent an improvement favouring the intervention group. $k$ represents the number of primary trials included in the analysis. If a review reported several effect sizes within each outcome domain, a composite was created and $k$ denotes the range of the number of primary trials that contributed to the effect estimate. AMSTAR $=$ a measurement tool to assess systematic reviews (max score 16); $\mathrm{OA}=$ older adults; $\mathrm{MCI}=$ mild cognitive impairment $\mathrm{PD}=$ Parkinson's disease.* total number of studies in review. 
in PD (cognitive training, I. H. Leung et al., 2015), two in stroke (cognitive training, das Nair et al., 2016; Loetscher \& Lincoln, 2013) and one in mixed MCI and dementia (cognitive training, Hoefler, 2016). The majority found no significant difference between intervention and control groups on functional abilities. Two reviews with critically low AMSTAR ratings showed contrary results. Chandler et al. (2016) demonstrated a small and significant effect of mixed cognition-oriented treatments on ADL for MCI and Hoefler (2016) showed a small and significant effect of computerized cognitive training on ADL in mixed MCI and dementia.

\section{Caregiver Outcomes}

Four reviews assessed caregiver outcomes, all of which focused on caregivers of people with dementia. One review assessed cognitive stimulation (Woods et al., 2012), one focused on cognitive training (Bahar-Fuchs et al., 2019) and two were classified as mixed cognition-oriented treatments (Lee et al., 2019; Leung et al., 2017). No significant interventional effect was found on the overall caregiver outcomes (Fig. 4). A review with low confidence AMSTAR rating by Leung et al. (2017) reported a small and significant benefit of mixed cognition-oriented treatments with caregiver involvement on carer quality of life and caregiver depressive symptoms, but no significant benefits on caregiver burden, caregiver anxiety or carer to person-with-dementia relationship.

\section{Clinical Outcomes}

Two reviews reported meta-analytic results for clinical outcomes (Fig. 4). A moderate quality review showed a large and significant effect of cognitive training on dementia disease progression with Hedges' $g=1.07$ (0.59 to 1.55 ), however, authors stated that there was uncertainty in the estimate due to large heterogeneity and imprecision (Bahar-Fuchs et al., 2019).

\section{Behavioural and Psychological Symptoms of Dementia}

Three reviews reported meta-analytic results for the effects of cognitive stimulation (Kim et al., 2017; Woods et al., 2012) and cognitive training (Bahar-Fuchs et al., 2019) on behavioural and psychological symptoms in dementia. No significant benefits of the interventions were found (effect sizes ranging from -0.14 to 0.44 ).

\section{Meta-Regression}

As expected given substantial overlap of trials across reviews, heterogeneity within reviews $\left(\tau_{(2)}^{2}=0.009\right)$ was markedly larger than between reviews $\left(\tau_{(3)}^{2}=0.0004\right)$. Higher
AMSTAR score was associated with larger effect estimates for objective cognitive outcomes ( $\beta=0.011,95 \% \mathrm{CI} 0.002$ to $0.020, p=0.02, R_{(3)}^{2}=17 \%$, Fig. 5a). There was no evidence for association between effect estimates on cognitive outcomes and year of publication ( $\beta=0.004,95 \% \mathrm{CI}-0.009$ to $\left.0.017, p=0.50, R_{(3)}^{2}=5 \%\right)$ or the number of primary trials included in reviews ( $\beta=0.002,95 \%$ CI -0.001 to $0.004, p=$ $0.26, R_{(2)}^{2}=2 \%, R_{(3)}^{2}=34 \%$, Fig. $5 \mathrm{~b}$ and $\left.\mathrm{c}\right)$.

\section{Discussion}

The aim of this overview was to synthesize the available evidence from systematic reviews with meta-analyses investigating the efficacy of the three main types of cognition-oriented treatments for older adults with or without cognitive decline on cognitive and non-cognitive outcomes, while also taking into account the methodological quality of published reviews. We identified 51 reviews that met our inclusion criteria, 46 of which were included in the quantitative synthesis of results.

\section{Summary of Main Results}

There was consistent evidence to support the use of cognitive training for improving cognitive performance in healthy older adults, MCI and PD. For people with dementia, results from published meta-analyses were heterogeneous, however, the most recent and comprehensive review (Bahar-Fuchs et al., 2019) demonstrated moderate-quality evidence for cognitive training improving cognitive performance also in this population. Thus, the potential for cognitive training to improve cognitive outcomes has been demonstrated across the spectrum of cognitive health, from healthy older adults to dementia. We further found moderate-quality evidence to support the use of cognitive stimulation for improving cognitive performance for people with dementia. The mean effect estimates for cognitive training and cognitive stimulation across the different populations suggest modest efficacy. For stroke, we found that metaanalyses of cognitive training interventions targeting a specific cognitive domain, such as memory (das Nair et al., 2016) and attention (Loetscher \& Lincoln, 2013; Virk et al., 2015) showed no significant effect, however, results were imprecise and based on a small number of primary trials. In contrast, there was evidence from a moderate-quality review including 22 primary trials covering a variety of cognitive remediation techniques (Rogers et al., 2018), to support the use of cognition-oriented treatments to improve cognitive performance following stroke.

The evidence for cognition-oriented treatments to reduce subjective cognitive complaints was inconsistent, and although several reviews reported benefits on subjective cognitive outcomes, the confidence ratings for these reviews were 


\begin{tabular}{|c|c|c|c|c|c|c|}
\hline Population & Study & Subjective cognitive outcomes & Hedges' g $(95 \% \mathrm{Cl})$ & $k$ & & AMSTAR \\
\hline Healthy $O A$ & $\begin{array}{l}\text { Metternich } 2010 \\
\text { Kelly } 2014 \\
\text { Bhome } 2018 \\
\text { Hudes } 2019\end{array}$ & $\stackrel{\longmapsto}{\longmapsto \longmapsto}$ & $\begin{array}{l}0.27(0.01 \text { to } 0.52) \\
0.19(0.06 \text { to } 0.32) \\
0.06(-0.12 \text { to } 0.24) \\
0.66(0.23 \text { to } 1.08)\end{array}$ & $\begin{array}{c}3-4 \\
2-3 \\
6 \\
5-10\end{array}$ & $\begin{array}{l}6 \\
9 \\
8 \\
7\end{array}$ & $\begin{array}{l}\text { Critically low } \\
\text { Critically low } \\
\text { Low } \\
\text { Low }\end{array}$ \\
\hline $\mathrm{MCl}$ & Chandler 2016 & $\vdots \longmapsto$ & $0.37(0.16$ to 0.58$)$ & $26^{\star}$ & 8.5 & Critically low \\
\hline Dementia & Bahar-Fuchs 2019 & $\vdots$ & $0.25(-0.76$ to 1.25$)$ & 2 & 14 & Moderate \\
\hline Stroke & $\begin{array}{l}\text { Loetscher } 2013 \\
\text { das Nair } 2016\end{array}$ & $\longrightarrow$ & $\begin{array}{l}0.53(-0.02 \text { to } 1.08) \\
0.36(0.08 \text { to } 0.64)\end{array}$ & $\begin{array}{l}2 \\
7\end{array}$ & $\begin{array}{l}13 \\
12\end{array}$ & $\begin{array}{l}\text { Low } \\
\text { Low }\end{array}$ \\
\hline \multirow[t]{2}{*}{ Mixed } & $\begin{array}{l}\text { Yang } 2018 \\
\text { Floyd } 1997\end{array}$ & $\vdots \longmapsto \longmapsto$ & $\begin{array}{l}0.67(0.41 \text { to } 0.93) \\
0.32(0.16 \text { to } 0.47)\end{array}$ & $\begin{array}{c}8 \\
6-11\end{array}$ & $\begin{array}{c}6 \\
5.5\end{array}$ & $\begin{array}{l}\text { Low } \\
\text { Critically low }\end{array}$ \\
\hline & & 0.0 & & & & \\
\hline
\end{tabular}

\begin{tabular}{|c|c|c|c|c|c|c|}
\hline Population & Study & Psychosocial outcomes & Hedges' g $(95 \% \mathrm{Cl})$ & $k$ & & AMSTAR \\
\hline Healthy $O A$ & $\begin{array}{l}\text { Metternich } 2010 \\
\text { Bhome } 2018 \\
\text { Hudes } 2019\end{array}$ & $\longmapsto$ & $\begin{array}{l}0.02(-0.47 \text { to } 0.52) \\
0.25(0.05 \text { to } 0.45) \\
0.42(0.09 \text { to } 0.74)\end{array}$ & $\begin{array}{c}2 \\
6 \\
3-5\end{array}$ & $\begin{array}{l}6 \\
8 \\
7\end{array}$ & $\begin{array}{l}\text { Critically low } \\
\text { Low } \\
\text { Low }\end{array}$ \\
\hline $\mathrm{MCl}$ & $\begin{array}{l}\text { Chandler } 2016 \\
\text { Hill } 2017 \\
\text { Gates 2019b }\end{array}$ & 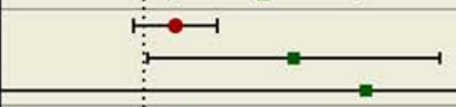 & $\begin{array}{l}0.11(-0.04 \text { to } 0.26) \\
0.52(0.01 \text { to } 1.03) \\
0.77(-0.52 \text { to } 2.06)\end{array}$ & $\begin{array}{c}26^{*} \\
8 \\
3\end{array}$ & $\begin{array}{c}8.5 \\
12 \\
12.5\end{array}$ & $\begin{array}{l}\text { Critically low } \\
\text { Moderate } \\
\text { Low }\end{array}$ \\
\hline Dementia & $\begin{array}{l}\text { Cooper } 2012 \\
\text { Woods } 2012 \\
\text { Folkerts } 2017 \\
\text { Kim } 2017 \\
\text { Hill } 2017 \\
\text { Bahar-Fuchs } 2019\end{array}$ & & $\begin{array}{l}0.16(-0.36 \text { to } 0.68) \\
0.27(0.00 \text { to } 0.55) \\
0.33(-0.10 \text { to } 0.76) \\
0.29(0.02 \text { to } 0.56) \\
0.60(-0.05 \text { to } 1.25) \\
0.34(-0.28 \text { to } 0.96)\end{array}$ & $\begin{array}{c}2 \\
4-5 \\
2 \\
4-6 \\
6 \\
5-8\end{array}$ & $\begin{array}{c}7 \\
10.5 \\
10.5 \\
7 \\
12 \\
14\end{array}$ & $\begin{array}{l}\text { Low } \\
\text { Moderate } \\
\text { Low } \\
\text { Critically low } \\
\text { Moderate } \\
\text { Moderate }\end{array}$ \\
\hline PD & Leung 2015 & 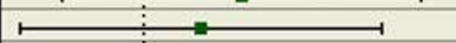 & $0.20(-0.43$ to 0.83$)$ & $3-5$ & 10 & Moderate \\
\hline Stroke & $\begin{array}{l}\text { Loetscher } 2013 \\
\text { das Nair } 2016\end{array}$ & $\longmapsto$ & $\begin{array}{l}0.02(-0.36 \text { to } 0.39) \\
-0.07(-0.35 \text { to } 0.21)\end{array}$ & $\begin{array}{c}2-3 \\
3\end{array}$ & $\begin{array}{l}13 \\
12\end{array}$ & $\begin{array}{l}\text { Low } \\
\text { Low }\end{array}$ \\
\hline \multirow[t]{2}{*}{ Mixed } & $\begin{array}{l}\text { Yang } 2018 \\
\text { Floyd } 1997 \\
\text { Pinquart } 2001 \\
\text { Hoefler } 2016\end{array}$ & $\stackrel{\longmapsto}{\longmapsto}$ & $\begin{array}{l}0.51(0.24 \text { to } 0.78) \\
0.08(-0.17 \text { to } 0.32) \\
0.26(-0.14 \text { to } 0.66) \\
0.36(0.09 \text { to } 0.63)\end{array}$ & $\begin{array}{c}10 \\
3-4 \\
2-9 \\
6\end{array}$ & $\begin{array}{c}6 \\
5.5 \\
1.5 \\
2.5\end{array}$ & $\begin{array}{l}\text { Low } \\
\text { Critically low } \\
\text { Critically low } \\
\text { Critically low }\end{array}$ \\
\hline & & 0.5 & & & & \\
\hline
\end{tabular}

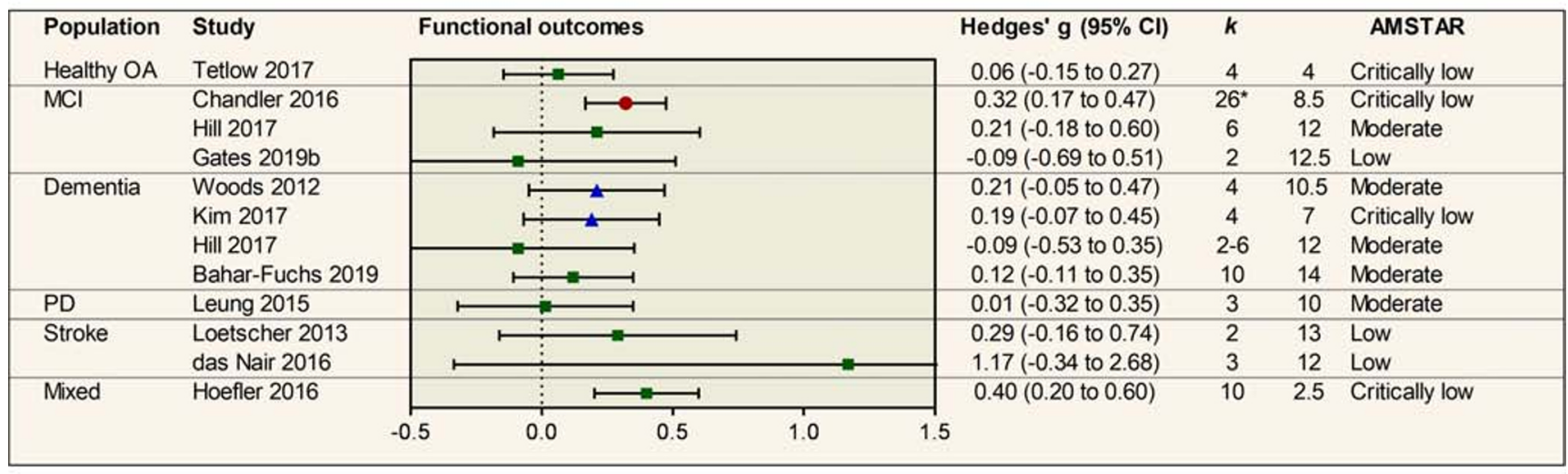

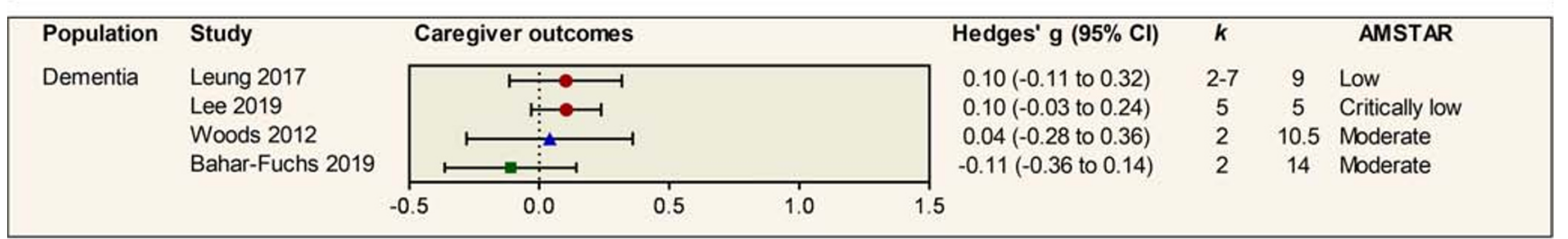

\begin{tabular}{|c|c|c|c|c|c|c|c|c|c|c|}
\hline \multirow{2}{*}{$\begin{array}{l}\text { Population } \\
\text { Dementia }\end{array}$} & \multirow{2}{*}{$\begin{array}{l}\text { Study } \\
\text { Bahar-Fuchs } 2019\end{array}$} & \multicolumn{5}{|c|}{ Clinical outcomes } & \multirow{2}{*}{$\begin{array}{c}\text { Hedges' } \mathbf{g}(95 \% \mathrm{Cl}) \\
1.07(0.59 \text { to } 1.55)\end{array}$} & \multirow{2}{*}{$\begin{array}{l}k \\
5\end{array}$} & \multirow{2}{*}{\multicolumn{2}{|c|}{$\begin{array}{l}\text { AMSTAR } \\
14 \text { Moderate }\end{array}$}} \\
\hline & & & $\vdots$ & & $\longrightarrow$ & & & & & \\
\hline \multirow[t]{2}{*}{ Mixed } & Hoefler 2016 & & $\vdots$ & & & & $0.13(-0.18$ to 0.44$)$ & 5 & 2.5 & Critically low \\
\hline & & -0.5 & 0.0 & 0.5 & 1.0 & 1.5 & & & & \\
\hline
\end{tabular}


Fig. 4 Pooled results of meta-analyses investigating subjective cognitive, psychosocial, functional, caregiver and clinical outcomes of cognitionoriented treatments (COT) in older adults. Positive values represent an improvement favouring the intervention group. $k$ represents the number of primary trials included in the analysis. If a review reported several effect sizes within each outcome domain, a composite was created and $k$ denotes the range of the number of primary trials that contributed to the effect estimate. AMSTAR = a measurement tool to assess systematic reviews (max score 16$)$; $\mathrm{OA}=$ older adults; $\mathrm{MCI}=$ mild cognitive impairment; $\mathrm{PD}=$ Parkinson's disease. * total number of studies in review.

low or critically low. For psychosocial outcomes, the majority of the reviews found no significant effect. Nevertheless, there was moderate-quality evidence from two reviews to support the efficacy of cognitive training to improve psychosocial functioning in MCI (Hill et al., 2017), and of cognitive stimulation to improve psychosocial functioning in dementia (Woods et al., 2012). There was no convincing evidence supporting the efficacy of cognition-oriented treatments on functional abilities, caregiver outcomes, clinical disease progression or behavioural and psychological symptoms of dementia. Overall, the quality of the evidence for subjective cognition and non-cognitive outcomes was restricted by methodological issues, such as methodological limitations of reviews, a small number of primary trials investigating these outcomes, and imprecise effect estimates.

\section{Overall Completeness of the Evidence}

Of the 46 meta-analyses included in this overview, 20 (43\%) reported pooled effect estimates for cognitive training in healthy older adults. Although some of these meta-analyses covered different subtypes of cognitive training approaches and outcome domains, it is still noteworthy that so many meta-analyses addressing the same or closely related questions have been published. Additionally, even though the overall number of primary trials published increases with time, there is no clear indication that the number of trials included in the meta-analyses has increased by year of publication (Fig. 3). Cognitive training has also been thoroughly investigated across older adult populations with cognitive decline, as we identified multiple meta-analyses on cognitive training for MCI, dementia, PD and stroke. For cognitive stimulation, meta-analytic results were only identified for people with dementia. Sherman et al. (2017) included cognitive stimulation in their review of cognitive interventions in MCI but did not identify any eligible trials. Finally, although a protocol for a meta-analysis of cognitive rehabilitation for people with dementia was recently published (Kudlicka et al., 2019), no current reviews were identified that reported meta-analytic results for cognitive rehabilitation, and it was recently concluded that the number of cognitive rehabilitation trials for Alzheimer's disease (Oltra-Cucarella et al., 2018) and other progressive neurodegenerative disorders (Clare et al., 2018) is still limited. Since cognitive rehabilitation is the cognition-oriented treatment approach that is most directly targeted towards producing functional change (Bahar-Fuchs et al., 2019), future trials exploring the effects of cognitive rehabilitation on functional abilities is an important area of investigation, especially considering the weak evidence for functional improvement following cognitive training and cognitive stimulation.

Cognitive performance was the primary outcome in most meta-analyses, and results for subjective cognition and noncognitive outcomes were more sparsely reported. Notably, only two reviews reported meta-analytic results for clinical outcomes, such as disease progression. This could be partly due to the fact that we only included post intervention results and that these outcomes are more appropriate to evaluate at follow-up assessments. However, the most recent Cochrane review on cognitive training in dementia identified only two primary trials that reported results for disease progression at follow-up assessments 3 to 12 months post treatment (BaharFuchs et al., 2019). This corroborates the inference that despite their importance - clinical outcomes are rarely assessed in primary trials. Moreover, although several reviews reported meta-analytic results for subjective cognition, psychosocial and functional outcomes, the pooled effect estimates were generally based on a small number of primary trials and, within these broad categories, a diverse set of outcomes and instruments had been employed. The fact that clinically relevant outcomes are assessed infrequently and with heterogeneous measures has previously been highlighted (Harrison, Noel-Storr, Demeyere, Reynish, \& Quinn, 2016; Lees, Fearon, Harrison, Broomfield, \& Quinn, 2012) and attempts have been made to increase harmonisation (Webster et al., 2017), which seems imperative in order to improve synthesis efforts in the field of cognition-oriented treatments.

\section{Methodological Quality of Included Meta-Analyses}

The methodological quality of included meta-analyses was highly variable, and we found no clear evidence that methodological standards in reviews have improved in the past 10 years. Strengths included using PICO as an organising framework for formulating the research question, conducting a comprehensive literature search, reporting conflicts of interest and discussing the impact of heterogeneity on the results. However, there were also important limitations. Most reviews did not report the sources of funding for included studies, which could be argued to be particularly important in the field of cognitive training which has been criticized for commercial interests (Stanford Center on Longevity, 2014). Additionally, the majority of reviews did not have a preregistered protocol. Protocol preregistration is important to mitigate bias in the review process (Stewart, Moher, \& Shekelle, 2012) and we 

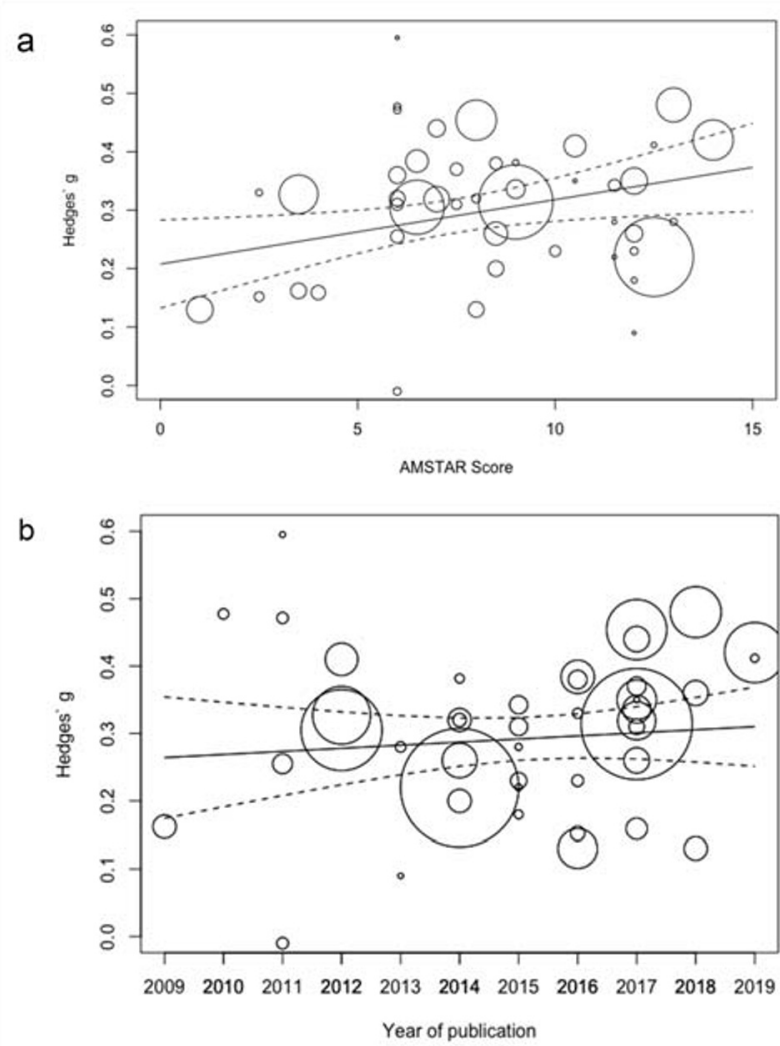

C

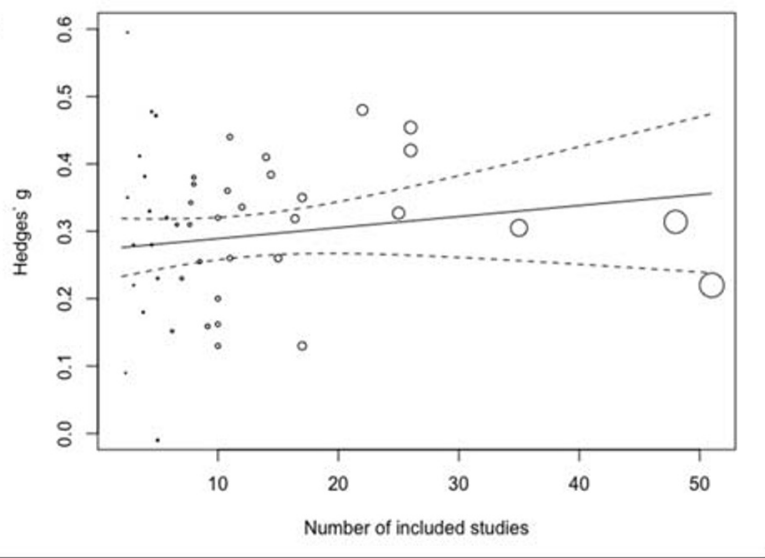

Fig. 5 Association between objective cognition effect size and (a) AMSTAR score, (b) year of publication and (c) number of included studies. Circle size refer to the number of included studies. Two extreme effect sizes (Folkerts et al., 2017; Gates, Rutjes, et al., 2019) were omitted from the scatterplots. AMSTAR = a measurement tool to assess systematic reviews.

found that referral to a protocol was associated with higher quality score, over and above the one point given for protocol preregistration. Other concerns were that the majority of reviews did not provide a list of excluded studies and justify their exclusion, nor did they assess the potential impact of risk of bias on the results.

Notably, more than half of the reviews included in this overview received a critically low confidence rating using the AMSTAR tool. This could in part be attributed to the fact that we used fairly wide inclusion criteria and did not restrict our overview to meta-analyses that included only RCTs or to more recent publications. As might be expected, older metaanalyses did not comply to modern standards for metaanalytic methods and reporting and consequently obtained a lower AMSTAR score. Nevertheless, the majority of included meta-analyses were published in the past 10 years and for these, no evidence was found to suggest that review quality improved by year of publication. We further found that higher AMSTAR score was associated with larger cognitive effect sizes. This suggests that further investigation into the relation between the methodological conduct of reviews and their corresponding meta-analytic results might be warranted. Caution has been raised that the rapid increase in published systematic reviews and meta-analyses in the past decades - in some areas even outpacing the number of published RCTs (Niforatos, Weaver, \& Johansen, 2019) - has brought about redundant and potentially flawed meta-analyses (Ioannidis, 2016). The results from this overview suggest that a critical appraisal and the implementation of mechanisms to reduce the rate of duplication and redundancy is warranted also in the field of cognition-oriented treatments in older adults.

\section{Implications for Research}

This overview highlights that despite the relatively large evidence-base in the field of cognition-oriented treatments for older adults, there are also areas in which more research is needed. Since we only identified meta-analyses on cognitive stimulation for people with dementia, a future direction for primary trials and subsequent evidence synthesis is to explore the efficacy of cognitive stimulation in other age-related conditions associated with cognitive decline. The effects of cognitive rehabilitation remains largely unexplored, however, a Cochrane review on cognitive rehabilitation in dementia is underway (Kudlicka et al., 2019). Future research on cognitive rehabilitation in other conditions, such as PD and MCI, could also be of value.

It is clear that understanding of the clinical relevance of cognition-oriented treatments is dependent on identifying which outcomes are clinically relevant for patients and caregivers and including these in a more consistent way in future trials. Furthermore, to increase confidence in the available evidence, future meta-analyses should be conducted with rigorous methodological standards and in accordance with available guidelines. Critical areas of improvement include using publicly available and preregistered protocols to reduce the risk of bias in the review process, reporting sources of funding for primary trials, and assessing the impact of risk of bias on the meta-analytic results to confirm their reliability.

Given the wealth of evidence for cognition-oriented treatments improving cognitive outcomes in older adults, evidence 
synthesis in the field may be most valuable by gradually changing its main focus from questions of mere efficacy to investigating what makes interventions more effective for specific cognition-oriented treatments and target populations. This will require more comprehensive methods than those currently employed in the field, but are nevertheless reasonably developed and have already proved as practice-changing in other clinical fields.

First, the availability of multiple and often overlapping outcome measures within primary trials means that effect sizes are dependent and therefore cannot be simply pooled together in the same analysis. This challenge has so far been handled in various ways, most commonly by selecting a single outcome measure per analysis or by creating composites based on simple means of all available measures of a given outcome. Designs in which data is pooled only when the same outcome measure was used appear to make sense psychometrically, but given limited overlap of outcome measures across studies, this is likely to lead to a large number of small and underpowered analyses, leading to both type I (multiple comparisons) and type II (small sample) errors. Analyses of composite scores of multiple outcome measures might avoid this problem, but may overestimate within-study variance and thus underestimate between-study heterogeneity. Multivariate and multilevel approaches are likely to be more efficient alternatives, as they account for within- and between-study variance and thus allow not only to control for dependency among effect sizes but also to investigate potential sources of variance in each level (for a review, see Cheung, 2019).

Second, investigations of heterogeneity (mixed-effects subgroup analyses and meta-regressions) can be expanded to incorporate network meta-analysis approaches. In addition to attempts to compare different cognition-oriented treatment approaches (e.g., Liang et al., 2018), it may be possible to use component network meta-analysis (Pompoli et al., 2018), which allows for different intervention components to be dismantled and compared in order to identify the optimal combination of intervention ingredients or techniques. In complex interventions such as cognition-oriented treatments, this approach could lead to a better understanding of the most (or least) essential components, which could have important theoretical and clinical implications.

Finally, there is a clear need to understand who is more likely to adhere to and benefit from specific interventions. Individual participant data meta-analyses have the potential to produce fine-grained evidence of the interaction between personal and intervention design factors but are yet to be conducted in the field. As with other more advanced and arguably more clinically informative methods, these types of metaanalyses will require more expansive eligibility criteria aiming to capture clinical and methodological heterogeneity, rather than more restrictive approaches.

\section{Implications for Practice}

The evidence in this overview suggests that cognitionoriented treatments lead to a modest improvement in cognitive performance on standardized measures. Cognition-oriented treatments may be beneficial for subjective cognition and psychosocial functioning, however, the available evidence for benefit was inconsistent and generally of low quality. No convincing evidence suggests that cognition-oriented treatments are associated with benefits on functional abilities, clinical disease progression, caregiver outcomes or behavioural and psychological symptoms of dementia. For many clinically relevant outcomes, the confidence in the available evidence is low, suggesting that the observed effects may change as a result of further, high-quality research. Placing the results from this overview in the context of available treatments for age-associated cognitive decline, the observed benefits of cognition-oriented treatments on cognition are in line with those reported for pharmacological treatments for dementia (Birks \& Harvey, 2018), but without, or very rare, adverse effects. Taken together, the available evidence suggests that cognition-oriented treatments are an acceptable approach to maintain cognitive health in old age, while the trade-off between conducting cognition-oriented treatments and participating in other potentially engaging activities also needs to be considered.

\section{Strengths and Limitations}

This overview followed a preregistered protocol and was conducted in accordance with established criteria for systematic reviews. We made efforts to categorize the intervention approach of each included meta-analysis using established definitions (Clare \& Woods, 2004) and used a rigorous and detailed instrument for quality assessment (Shea et al., 2017). A broad set of inclusion criteria were applied, in order to identify and synthesize the available evidence on the different types of cognition-oriented treatments on cognitive and non-cognitive outcomes across older adult populations. Thus, this overview provides a comprehensive summary of published meta-analyses in the field, as well as their methodological quality.

Some limitations also need to be addressed. First, the distinction between the different cognition-oriented treatment approaches is not uncomplicated. As has previously been recognized (Bahar-Fuchs et al., 2019; Tardif \& Simard, 2011) the different types of cognition-oriented treatments are sometimes used interchangeably in the literature and interventional elements belonging to the different categories can overlap within trials. In our overview, three meta-analyses (das Nair et al., 2016; Loetscher \& Lincoln, 2013; Virk et al., 2015) were described as cognitive rehabilitation by review authors, but reclassified as cognitive 
training in order to be consistent with definitions provided by Clare and Woods (2004). This definition of cognitive rehabilitation emphasizes an individualized approach based on collaborative goal-setting. However, other definitions have also been used, particularly in the field of stroke rehabilitation (Cicerone et al., 2000). Second, since many of the meta-analyses focused on the same intervention or population combination, there is overlap in the primary trials included. This can give a false impression of agreement across meta-analyses and it is important to emphasize that the strength of the evidence is not related to the number of published meta-analyses, but to their comprehensiveness and methodological quality. Third, we used broad intervention and outcome categories and the specific interventions and outcomes included in each category were heterogeneous. Thus, a more detailed investigation of the efficacy of different sub-types of cognition-oriented treatments on specific outcome sub-domains is likely to be informative. However, such an approach was beyond the scope of this overview, as our primary objective was to provide a broad overview of the field. For this reason, we also chose to include meta-analyses that were based on both RCTs and non-RCTs. This could arguably introduce additional sources of bias, since the quality of the evidence from a meta-analysis is always dependent on the methodological quality of included primary trials. However, the inclusion of non-RCTs is accounted for in the quality ratings and consequently also in the overall interpretation of the results. Finally, this overview was restricted to meta-analyses. Thus, this excludes other potentially relevant evidence from systematic reviews without meta-analysis and primary trials.

\section{Conclusions}

This systematic overview showed that cognition-oriented treatments are efficacious in improving cognitive performance in older adults with and without cognitive decline. Whether these effects translate into improvements in clinically meaningful outcomes remains unclear, due to the scarcity of high-quality evidence for outcomes of potential clinical relevance. To establish the clinical usefulness, or lack thereof, of cognition-oriented treatments, an important avenue for future trials is to include relevant noncognitive outcomes in a more consistent way. We encourage future trials on the efficacy of cognitive rehabilitation across older adult populations, and on cognitive stimulation for non-demented older adults with cognitive decline. For meta-analyses in the field, there is a need for better adherence to methodological standards, and protocol preregistration should be encouraged.
Acknowledgements HMG was supported by grants from the Graduate School in Population Dynamics and Public Policy, Umeå university and the Helge Ax:son Jonson's Foundation. AL and ABF are supported by dementia research fellowships from the National Health and Medical Research Council of Australia (GNT1108520, GNT1135605).

Funding Information Open access funding provided by Umea University.

\section{Compliance with Ethical Standards}

Conflict of Interest $\mathrm{ABF}$ and JS are authors on one of the reviews included in this overview, $\mathrm{HH}$ is an author of two reviews, and $\mathrm{AL}$ is an author on three reviews included in this overview. They were not involved in the quality assessment of these reviews.

Open Access This article is licensed under a Creative Commons Attribution 4.0 International License, which permits use, sharing, adaptation, distribution and reproduction in any medium or format, as long as you give appropriate credit to the original author(s) and the source, provide a link to the Creative Commons licence, and indicate if changes were made. The images or other third party material in this article are included in the article's Creative Commons licence, unless indicated otherwise in a credit line to the material. If material is not included in the article's Creative Commons licence and your intended use is not permitted by statutory regulation or exceeds the permitted use, you will need to obtain permission directly from the copyright holder. To view a copy of this licence, visit http://creativecommons.org/licenses/by/4.0/.

\section{References}

Aarsland, D., Creese, B., Politis, M., Chaudhuri, K. R., Ffytche, D. H., Weintraub, D., \& Ballard, C. (2017). Cognitive decline in Parkinson disease. Nature Reviews Neurology, 13(4), 217-231. https://doi.org/ 10.1038/nrneurol.2017.27

Alves, J., Magalhaes, R., Thomas, R. E., Goncalves, O. F., Petrosyan, A., \& Sampaio, A. (2013). Is there evidence for cognitive intervention in Alzheimer disease? A systematic review of efficacy, feasibility, and cost-effectiveness. Alzheimer Disease \& Associated Disorders, 27(3), 195-203. https://doi.org/10.1097/WAD.0b013e31827bda55

Bahar-Fuchs, A., Clare, L., \& Woods, B. (2019). Cognitive training and cognitive rehabilitation for mild to moderate Alzheimer's disease and vascular dementia. Cochrane Database of Systematic Reviews, 6, CD003260. https://doi.org/10.1002/14651858.CD003260.pub2

Bahar-Fuchs, A., Martyr, A., Goh, A. M., Sabates, J., \& Clare, L. (2019). Cognitive training for people with mild to moderate dementia. Cochrane Database of Systematic Reviews, 3, CD013069. https:// doi.org/10.1002/14651858.CD013069.pub2

Bhome, R., Berry, A. J., Huntley, J. D., \& Howard, R. J. (2018). Interventions for subjective cognitive decline: Systematic review and meta-analysis. BMJ Open, 8(7), e021610. https://doi.org/10. 1136/bmjopen-2018-021610

Birks, J. S., \& Harvey, R. J. (2018). Donepezil for dementia due to Alzheimer's disease. Cochrane Database of Systematic Reviews, 6, CD001190. https://doi.org/10.1002/14651858.CD001190.pub3

Chandler, M. J., Parks, A. C., Marsiske, M., Rotblatt, L. J., \& Smith, G. E. (2016). Everyday impact of cognitive interventions in mild cognitive impairment: A systematic review and meta-analysis. Neuropsychology Review, 26(3), 225-251. https://doi.org/10.1007/ s11065-016-9330-4 
Cheung, M. W. (2014). metaSEM: An R package for meta-analysis using structural equation modeling. Frontiers in Psychology, 5, 1521. https://doi.org/10.3389/fpsyg.2014.01521

Cheung, M. W. (2019). A guide to conducting a meta-analysis with nonindependent effect sizes. 1-10.:https://doi.org/10.1007/s11065-01909415-6

Chiu, H. L., Chu, H., Tsai, J. C., Liu, D., Chen, Y. R., Yang, H. L., \& Chou, K. R. (2017). The effect of cognitive-based training for the healthy older people: A meta-analysis of randomized controlled trials. PLoS One, 12(5), e0176742. https://doi.org/10.1371/journal. pone. 0176742

Cicerone, K. D., Dahlberg, C., Kalmar, K., Langenbahn, D. M., Malec, J. F., Bergquist, T. F., ... Morse, P. A. (2000). Evidence-based cognitive rehabilitation: Recommendations for clinical practice. Archives of Physical Medicine and Rehabilitation, 81(12), 1596-1615. https://doi.org/10.1053/apmr.2000.19240

Clare, L., Teale, J. C., Toms, G., Kudlicka, A., Evans, I., Abrahams, S., ... Thompson-Coon, J. (2018). Cognitive rehabilitation, self-management, psychotherapeutic and caregiver support interventions in progressive neurodegenerative conditions: A scoping review. NeuroRehabilitation, 43(4), 443-471. https://doi.org/10.3233/ NRE-172353

Clare, L., \& Woods, R. T. (2004). Cognitive training and cognitive rehabilitation for people with early-stage Alzheimer's disease: A review. Neuropsychological Rehabilitation, 14(4), 385-401. https://doi.org/ 10.1080/09602010443000074

Cognitive Training Data. (2014). Open letter response to the Stanford Center on longevity. Retrieved October 2019 from https://www. cognitivetrainingdata.org/the-controversy-does-brain-trainingwork/response-letter/

Cooper, C., Mukadam, N., Katona, C., Lyketsos, C. G., Ames, D., Rabins, P., ... Livingston, G. (2012). Systematic review of the effectiveness of non-pharmacological interventions to improve quality of life of people with dementia. International Psychogeriatrics, 24(6), 856-870. https://doi.org/10.1017/S1041610211002614

Cooper, H., \& Koenka, A. C. (2012). The overview of reviews: Unique challenges and opportunities when research syntheses are the principal elements of new integrative scholarship. American Psychologist, 67(6), 446-462. https://doi.org/10.1037/a0027119

Cumming, T. B., Marshall, R. S., \& Lazar, R. M. (2013). Stroke, cognitive deficits, and rehabilitation: Still an incomplete picture. International Journal of Stroke, 8(1), 38-45. https://doi.org/10. 1111/j.1747-4949.2012.00972.x

das Nair, R., Cogger, H., Worthington, E., \& Lincoln, N. B. (2016). Cognitive rehabilitation for memory deficits after stroke. Cochrane Database of Systematic Reviews, 9, CD002293. https://doi.org/10. 1002/14651858.CD002293.pub3

Deary, I. J., Corley, J., Gow, A. J., Harris, S. E., Houlihan, L. M., Marioni, R. E., ... Starr, J. M. (2009). Age-associated cognitive decline. British Medical Bulletin, 92(1), 135-152. https://doi.org/10.1093/ bmb/ldp033

Floyd, M., \& Scogin, F. (1997). Effects of memory training on the subjective memory functioning and mental health of older adults: A meta-analysis. Psychology and Aging, 12(1), 150-161.

Folkerts, A. K., Roheger, M., Franklin, J., Middelstadt, J., \& Kalbe, E. (2017). Cognitive interventions in patients with dementia living in long-term care facilities: Systematic review and meta-analysis. Archives of Gerontology and Geriatrics, 73, 204-221. https://doi. org/10.1016/j.archger.2017.07.017

Gates, N. J., Rutjes, A. W., Di Nisio, M., Karim, S., Chong, L. Y., March, E., ... Vernooij, R. W. (2019). Computerised cognitive training for maintaining cognitive function in cognitively healthy people in late life. Cochrane Database of Systematic Reviews, 3, CD012277. https://doi.org/10.1002/14651858.CD012277.pub2

Gates, N. J., Vernooij, R. W., Di Nisio, M., Karim, S., March, E., Martinez, G., \& Rutjes, A. W. (2019). Computerised cognitive training for preventing dementia in people with mild cognitive impairment. Cochrane Database of Systematic Reviews, 3, CD012279. https://doi.org/10.1002/14651858.CD012279.pub2

Gross, A. L., Parisi, J. M., Spira, A. P., Kueider, A. M., Ko, J. Y., Saczynski, J. S., ... Rebok, G. W. (2012). Memory training interventions for older adults: A meta-analysis. Aging \& Mental Health, 16(6), 722-734. https://doi.org/10.1080/13607863.2012.667783

Harrison, J. K., Noel-Storr, A. H., Demeyere, N., Reynish, E. L., \& Quinn, T. J. (2016). Outcomes measures in a decade of dementia and mild cognitive impairment trials. Alzheimer's Research \& Therapy, 8(1), 48. https://doi.org/10.1186/s13195-016-0216-8

Hill, N. T., Mowszowski, L., Naismith, S. L., Chadwick, V. L., Valenzuela, M., \& Lampit, A. (2017). Computerized cognitive training in older adults with mild cognitive impairment or dementia: A systematic review and meta-analysis. American Journal of Psychiatry, 174(4), 329-340. https://doi.org/10.1176/appi.ajp. 2016.16030360

Hindin, S. B., \& Zelinski, E. M. (2012). Extended practice and aerobic exercise interventions benefit untrained cognitive outcomes in older adults: A meta-analysis. Journal of the American Geriatrics Society, 60(1), 136-141. https://doi.org/10.1111/j.1532-5415.2011.03761.x

Hoefler, C. E. (2016). Computer-based cognitive training and Alzheimer's disease: A meta-analysis. (doctoral dissertation), Saint Mary's University of Minnesota, Retrieved from ProQuest. (10164041)

Hudes, R., Rich, J. B., Troyer, A. K., Yusupov, I., \& Vandermorris, S. (2019). The impact of memory-strategy training interventions on participant-reported outcomes in healthy older adults: A systematic review and meta-analysis. Psychology and Aging, 34(4), 587-597. https://doi.org/10.1037/pag0000340

Huntley, J. D., Gould, R. L., Liu, K., Smith, M., \& Howard, R. J. (2015). Do cognitive interventions improve general cognition in dementia? A meta-analysis and meta-regression. BMJ Open, 5(4), e005247. https://doi.org/10.1136/bmjopen-2014-005247

Ioannidis, J. P. (2016). The mass production of redundant, misleading, and conflicted systematic reviews and meta-analyses. Milbank Quarterly, 94(3), 485-514. https://doi.org/10.1111/1468-0009. 12210

Karbach, J., \& Verhaeghen, P. (2014). Making working memory work: A meta-analysis of executive-control and working memory training in older adults. Psychological Science, 25(11), 2027-2037. https://doi. org/10.1177/0956797614548725

Karr, J. E., Areshenkoff, C. N., Rast, P., \& Garcia-Barrera, M. A. (2014). An empirical comparison of the therapeutic benefits of physical exercise and cognitive training on the executive functions of older adults: A meta-analysis of controlled trials. Neuropsychology, 28(6), 829-845. https://doi.org/10.1037/neu0000101

Kelly, M. E., Loughrey, D., Lawlor, B. A., Robertson, I. H., Walsh, C., \& Brennan, S. (2014). The impact of cognitive training and mental stimulation on cognitive and everyday functioning of healthy older adults: A systematic review and meta-analysis. Ageing Research Reviews, 15, 28-43. https://doi.org/10.1016/j.arr.2014.02.004

Kim, K., Han, J. W., So, Y., Seo, J., Kim, Y. J., Park, J. H., ... Kim, K. W. (2017). Cognitive stimulation as a therapeutic modality for dementia: A meta-analysis. Psychiatry Investigation, 14(5), 626-639. https://doi.org/10.4306/pi.2017.14.5.626

Kudlicka, A., Martyr, A., Bahar-Fuchs, A., Woods, B., \& Clare, L. (2019). Cognitive rehabilitation for people with mild to moderate dementia. Cochrane Database of Systematic Reviews, 7 , CD013388. https://doi.org/10.1002/14651858.CD013388

Kurz, A. F., Leucht, S., \& Lautenschlager, N. T. (2011). The clinical significance of cognition-focused interventions for cognitively impaired older adults: A systematic review of randomized controlled trials. International Psychogeriatrics, 23(9), 1364-1375. https://doi. org/10.1017/S1041610211001001 
Lampit, A., Hallock, H., \& Valenzuela, M. (2014). Computerized cognitive training in cognitively healthy older adults: A systematic review and meta-analysis of effect modifiers. PLoS Medicine, 11(11), e1001756. https://doi.org/10.1371/journal.pmed.1001756

Lawrence, B. J., Gasson, N., Bucks, R. S., Troeung, L., \& Loftus, A. M. (2017). Cognitive training and noninvasive brain stimulation for cognition in Parkinson's disease: A meta-analysis. Neurorehabiliation and Neural Repair, 31(7), 597-608. https:// doi.org/10.1177/1545968317712468

Lee, M., Ryoo, J. H., Chung, M., Anderson, J. G., Rose, K., \& Williams, I. C. (2019). Effective interventions for depressive symptoms among caregivers of people with dementia: A systematic review and metaanalysis. Dementia, Epub, (Jan 12). https://doi.org/10.1177/ 1471301218822640

Lees, R., Fearon, P., Harrison, J. K., Broomfield, N. M., \& Quinn, T. J. (2012). Cognitive and mood assessment in stroke research: Focused review of contemporary studies. Stroke, 43(6), 1678-1680. https:// doi.org/10.1161/STROKEAHA.112.653303

Leung, I. H., Walton, C. C., Hallock, H., Lewis, S. J., Valenzuela, M., \& Lampit, A. (2015). Cognitive training in Parkinson disease: A systematic review and meta-analysis. Neurology, 85(21), 1843-1851. https://doi.org/10.1212/WNL.0000000000002145

Leung, P., Orgeta, V., \& Orrell, M. (2017). The effects on carer well-being of carer involvement in cognition-based interventions for people with dementia: A systematic review and meta-analysis. International Journal of Geriatric Psychiatry, 32(4), 372-385. https://doi.org/10.1002/gps.4654

Liang, J. H., Xu, Y., Lin, L., Jia, R. X., Zhang, H. B., \& Hang, L. (2018). Comparison of multiple interventions for older adults with Alzheimer disease or mild cognitive impairment: A PRISMAcompliant network meta-analysis. Medicine (Baltimore), 97(20), e10744. https://doi.org/10.1097/MD.0000000000010744

Liberati, A., Altman, D. G., Tetzlaff, J., Mulrow, C., Gotzsche, P. C., Ioannidis, J. P., ... Moher, D. (2009). The PRISMA statement for reporting systematic reviews and meta-analyses of studies that evaluate health care interventions: Explanation and elaboration. PLoS Medicine, 6(7), e1000100. https://doi.org/10.1371/journal.pmed. 1000100

Loetscher, T., \& Lincoln, N. B. (2013). Cognitive rehabilitation for attention deficits following stroke. Cochrane Database of Systematic Reviews, 5, CD002842. https://doi.org/10.1002/14651858. CD002842.pub2

Martin, M., Clare, L., Altgassen, A. M., Cameron, M. H., \& Zehnder, F. (2011). Cognition-based interventions for healthy older people and people with mild cognitive impairment. Cochrane Database of Systematic Reviews, 1, CD006220. https://doi.org/10.1002/ 14651858.CD006220.pub2

McKenzie, J. E., \& Brennan, S. E. (2017). Overviews of systematic reviews: Great promise, greater challenge. Systematic Reviews, 6(1), 185. https://doi.org/10.1186/s13643-017-0582-8

Melby-Lervag, M., \& Hulme, C. (2016). There is no convincing evidence that working memory training is effective: A reply to au et al. (2014) and Karbach and Verhaeghen (2014). Psychonomic Bulletin \& Review, 23(1), 324-330. https://doi.org/10.3758/s13423-015-0862$\mathrm{Z}$

Metternich, B., Kosch, D., Kriston, L., Harter, M., \& Hull, M. (2010). The effects of nonpharmacological interventions on subjective memory complaints: A systematic review and meta-analysis. Psychotherapy and Psychosomatics, 79(1), 6-19. https://doi.org/ 10.1159/000254901

Mewborn, C. M., Lindbergh, C. A., \& Stephen Miller, L. (2017). Cognitive interventions for cognitively healthy, mildly impaired, and mixed samples of older adults: A systematic review and metaanalysis of randomized-controlled trials. Neuropsychology Review, 27(4), 403-439. https://doi.org/10.1007/s11065-017-9350-8
Niforatos, J. D., Weaver, M., \& Johansen, M. E. (2019). Assessment of publication trends of systematic reviews and randomized clinical trials, 1995 to 2017. JAMA Internal Medicine, Epub July 30. https://doi.org/10.1001/jamainternmed.2019.3013

Nyberg, L., Lovden, M., Riklund, K., Lindenberger, U., \& Backman, L. (2012). Memory aging and brain maintenance. Trends in Cognitive Science, 16(5), 292-305. https://doi.org/10.1016/j.tics.2012.04.005

Olazaran, J., Reisberg, B., Clare, L., Cruz, I., Pena-Casanova, J., Del Ser, T., ... Muniz, R. (2010). Nonpharmacological therapies in Alzheimer's disease: A systematic review of efficacy. Dementia and Geriatric Cognitive Disorders, 30(2), 161-178. https://doi. org/10.1159/000316119

Oltra-Cucarella, J., Ferrer-Cascales, R., Clare, L., Morris, S. B., Espert, R., Tirapu, J., \& Sanchez-SanSegundo, M. (2018). Differential effects of cognition-focused interventions for people with Alzheimer's disease: A meta-analysis. Neuropsychology, 32(6), 664-679. https:// doi.org/10.1037/neu0000449

Papp, K. V., Walsh, S. J., \& Snyder, P. J. (2009). Immediate and delayed effects of cognitive interventions in healthy elderly: A review of current literature and future directions. Alzheimer's and Dementia, 5(1), 50-60. https://doi.org/10.1016/j.jalz.2008.10.008

Pinquart, M., \& Sörensen, S. (2001). How effective are psychotherapeutic and other psychosocial interventions with older adults? A meta-analysis. Journal of Mental Health and Aging, 7(2), 207-243.

Pompoli, A., Furukawa, T. A., Efthimiou, O., Imai, H., Tajika, A., \& Salanti, G. (2018). Dismantling cognitive-behaviour therapy for panic disorder: A systematic review and component network metaanalysis. Psychological Medicine, 48(12), 1945-1953. https://doi. org/10.1017/S0033291717003919

Rogers, J. M., Foord, R., Stolwyk, R. J., Wong, D., \& Wilson, P. H. (2018). General and domain-specific effectiveness of cognitive remediation after stroke: Systematic literature review and meta-analysis. Neuropsychology Review, 28(3), 285-309. https://doi.org/10. 1007/s11065-018-9378-4

Shao, Y. K., Mang, J., Li, P. L., Wang, J., Deng, T., \& Xu, Z. X. (2015). Computer-based cognitive programs for improvement of memory, processing speed and executive function during age-related cognitive decline: A meta-analysis. PLoS One, 10(6), e0130831. https:// doi.org/10.1371/journal.pone.0130831

Shea, B. J., Reeves, B. C., Wells, G., Thuku, M., Hamel, C., Moran, J., ... Henry, D. A. (2017). AMSTAR 2: A critical appraisal tool for systematic reviews that include randomised or non-randomised studies of healthcare interventions, or both. $B M J, 358, \mathrm{j} 4008$. https://doi. org/10.1136/bmj.j4008

Sherman, D. S., Mauser, J., Nuno, M., \& Sherzai, D. (2017). The efficacy of cognitive intervention in mild cognitive impairment (MCI): A meta-analysis of outcomes on neuropsychological measures. Neuropsychology Review, 27(4), 440-484. https://doi.org/10.1007/ s11065-017-9363-3

Sitzer, D. I., Twamley, E. W., \& Jeste, D. V. (2006). Cognitive training in Alzheimer's disease: A meta-analysis of the literature. Acta Psychiatrica Scandinavia, 114(2), 75-90. https://doi.org/10.1111/j. 1600-0447.2006.00789.x

Smart, C. M., Karr, J. E., Areshenkoff, C. N., Rabin, L. A., Hudon, C., Gates, N., ... Wesselman, L. (2017). Non-pharmacologic interventions for older adults with subjective cognitive decline: Systematic review, meta-analysis, and preliminary recommendations. Neuropsychology Review, 27(3), 245-257. https://doi.org/10.1007/ s11065-017-9342-8

Song, Y. W., Lee, J. S., \& Song, A. Y. (2016). Meta-analysis about cognitive intervention effect applied to dementia patients. NeuroRehabilitation, 39(2), 319-327. https://doi.org/10.3233/ NRE-161363

Stanford Center on Longevity. (2014). A consensus on the brain training industry from the scientific community. Retrieved July 2019 from 
http://longevity.stanford.edu/a-consensus-on-the-brain-trainingindustry-from-the-scientific-community-2/

Stewart, L., Moher, D., \& Shekelle, P. (2012). Why prospective registration of systematic reviews makes sense. Systematic Reviews, 1, 7. https://doi.org/10.1186/2046-4053-1-7

Tardif, S., \& Simard, M. (2011). Cognitive stimulation programs in healthy elderly: A review. International Journal of Alzheimer's Disease, 2011, 378934. https://doi.org/10.4061/2011/378934

Tetlow, A. M., \& Edwards, J. D. (2017). Systematic literature review and meta-analysis of commercially available computerized cognitive training among older adults. Journal of Cognitive Enhancement, 1(4), 559-575. https://doi.org/10.1007/s41465-017-0051-2

Toril, P., Reales, J. M., \& Ballesteros, S. (2014). Video game training enhances cognition of older adults: A meta-analytic study. Psychology and Aging, 29(3), 706-716. https://doi.org/10.1037/ a0037507

Verhaeghen, P., Marcoen, A., \& Goossens, L. (1992). Improving memory performance in the aged through mnemonic training: A metaanalytic study. Psychology and Aging, 7(2), 242-251. https://doi. org/10.1037/0882-7974.7.2.242

Viechtbauer, W. (2010). Conducting meta-analyses in R with the metafor package. Journal of Statistical Software, 36(3), 1-48.

Virk, S., Williams, T., Brunsdon, R., Suh, F., \& Morrow, A. (2015). Cognitive remediation of attention deficits following acquired brain injury: A systematic review and meta-analysis. NeuroRehabilitation, 36(3), 367-377. https://doi.org/10.3233/ NRE-151225

Wang, C., Yu, J. T., Wang, H. F., Tan, C. C., Meng, X. F., \& Tan, L. (2014). Non-pharmacological interventions for patients with mild cognitive impairment: A meta-analysis of randomized controlled trials of cognition-based and exercise interventions. Journal of Alzheimer's Disease, 42(2), 663-678. https://doi.org/10.3233/JAD140660

Wang, P., Liu, H. H., Zhu, X. T., Meng, T., Li, H. J., \& Zuo, X. N. (2016). Action video game training for healthy adults: A meta-analytic study. Frontiers in Psychology, 7, 907. https://doi.org/10.3389/ fpsyg.2016.00907

Webster, L., Groskreutz, D., Grinbergs-Saull, A., Howard, R., O'Brien, J. T., Mountain, G., ... Livingston, G. (2017). Core outcome measures for interventions to prevent or slow the progress of dementia for people living with mild to moderate dementia: Systematic review and consensus recommendations. PLoS One, 12(6), e0179521. https://doi.org/10.1371/journal.pone.0179521

Weicker, J., Villringer, A., \& Thone-Otto, A. (2016). Can impaired working memory functioning be improved by training? A meta-analysis with a special focus on brain injured patients. Neuropsychology, 30(2), 190-212. https://doi.org/10.1037/neu0000227

Weintraub, S., Wicklund, A. H., \& Salmon, D. P. (2012). The neuropsychological profile of Alzheimer disease. Cold Spring Harbor Perspectives in Medicine, 2(4), a006171. https://doi.org/10.1101/ cshperspect.a006171

Wilson, K. Y. (2008). The effectiveness of memory training programs in improving the subjective memory characteristics of healthy older adults with memory complaints: A meta-analysis. (doctoral dissertation), Marshall University, retrieved from Marshall digital scholar. (paper 165).

Woods, B., Aguirre, E., Spector, A. E., \& Orrell, M. (2012). Cognitive stimulation to improve cognitive functioning in people with dementia. Cochrane Database of Systematic Reviews, 2, CD005562. https://doi.org/10.1002/14651858.CD005562.pub2

Yang, H. L., Chan, P. T., Chang, P. C., Chiu, H. L., Sheen Hsiao, S. T., Chu, H., \& Chou, K. R. (2018). Memory-focused interventions for people with cognitive disorders: A systematic review and metaanalysis of randomized controlled studies. International Journal of Nursing Studies, 78, 44-51. https://doi.org/10.1016/j.jinurstu.2017. 08.005

Publisher's Note Springer Nature remains neutral with regard to jurisdictional claims in published maps and institutional affiliations. 


\section{University Library}

\section{- M I N E R VA \\ A gateway to Melbourne's research publications}

Minerva Access is the Institutional Repository of The University of Melbourne

Author/s:

Gavelin, HM;Lampit, A;Hallock, H;Sabates, J;Bahar-Fuchs, A

Title:

Cognition-Oriented Treatments for Older Adults: a Systematic Overview of Systematic Reviews

Date:

2020-04-07

Citation:

Gavelin, H. M., Lampit, A., Hallock, H., Sabates, J. \& Bahar-Fuchs, A. (2020).

Cognition-Oriented Treatments for Older Adults: a Systematic Overview of Systematic Reviews. NEUROPSYCHOLOGY REVIEW, 30 (2), pp.167-193. https://doi.org/10.1007/ s11065-020-09434-8.

Persistent Link:

http://hdl.handle.net/11343/246007

License:

CC BY 